\title{
The magnetic flux of the quiet Sun internetwork as observed with the Tenerife infrared polarimeter ${ }^{\star}$
}

\author{
C. Beck $^{1}$ and R. Rezaei ${ }^{2}$ \\ 1 Instituto de Astrofísica de Canarias (CSIC), Via Lactéa, 38205 La Laguna (Tenerife), Spain \\ e-mail: cbeck@iac.es \\ 2 Kiepenheuer-Institut für Sonnenphysik, Schöneckstr. 6, 79104 Freiburg, Germany \\ e-mail: rrezaei@kis.uni-freiburg.de
}

Received 26 Junuary 2009 / Accepted 14 April 2009

\begin{abstract}
Context. Observations made with the spectropolarimeter onboard the HINODE satellite have detected abundant horizontal magnetic fields in the internetwork quiet Sun.

Aims. We compare the results for the horizontal fields obtained at $630 \mathrm{~nm}$ with ground-based observations at $1.56 \mu \mathrm{m}$, where the sensitivity to magnetic fields is higher than in the visible.

Methods. We obtained 30-s integrated spectropolarimetric data of the quiet Sun on disc centre during a period of extremely stable and good seeing. The data have a rms noise in polarization of around $2 \times 10^{-4}$ of the continuum intensity. The low noise level allows the spectra to be inverted with the SIR code. We compare the inversion results with proxies to determine the magnetic flux.

Results. We confirm the presence of the horizontal fields in the quiet Sun internetwork as reported for the satellite data, including voids without linear polarization signal that extend over an area of a few granules. Voids in the circular polarization signal are only of granular scale. More than $60 \%$ of the surface show polarization signals of above four times the rms noise level. We find that the total magnetic flux contained in the more inclined to horizontal fields $\left(\gamma>45^{\circ}\right)$ is lower by a factor of around 2 than that of the less inclined fields. The proxies for flux determination are strongly affected by the thermodynamic state of the atmosphere, and hence, seem to be unreliable.

Conclusions. During spells of good seeing conditions, adaptive optics can render ground-based slit-spectrograph observations at a $70-\mathrm{cm}$ telescope equivalent to the seeing-free space-based data of half-meter class telescopes. We suggest that the difference in the ratio of horizontal to transversal flux between the ground-based infrared data and the satellite-based visible data is due to the different formation heights of the respective spectral lines. We emphasize that the true amount of magnetic flux cannot be derived directly from the spectra. For purely horizontal flux, one would need its vertical extension that has to be estimated by explicit modeling, using the observed spectra as boundary conditions, or be taken from MHD simulations. Time-series of the evolution of the magnetic flux and chromospheric diagnostics are needed to address its possible contribution to chromospheric heating.
\end{abstract}

Key words. Sun: magnetic fields - Sun: photosphere

\section{Introduction}

Recent observations of magnetic fields in quiet Sun regions acquired with the spectropolarimeter onboard the HINODE satellite (Kosugi et al. 2007) have detected abundant horizontal magnetic fields (Orozco Suárez et al. 2007; Lites et al. 2008, hereafter OR07 and LI08), whose existence had been predicted before on the basis of the mismatch of observed average magnetic flux between Zeeman and Hanle measurements by Trujillo Bueno et al. (2004). Studies of photospheric magnetic fields using Zeeman-sensitive spectral lines in the visible (VIS) wavelength range, however, suffer from a severe drawback that depends on the wavelength: the thermal broadening $(\propto \lambda)$ dominates the Zeeman splitting caused by the magnetic field $\left(\propto \lambda^{2}\right)$. For spectral lines in the near-infrared (IR), the relation is more favorable. The retrieval of magnetic field properties from spectra in the VIS is thus less reliable, because the lines are in the weakfield limit, which imposes severe limits (Martínez González et al. $2006 \mathrm{~b}, 2008 \mathrm{c})$. The separation of the oppositely polarized spectral components is not proportional to the field strength, and

* Appendices A and B are only available in electronic form at http://www. aanda.org both field strength and magnetic flux can influence the amplitude of the polarization signal in similar ways. Observations of solar magnetic fields commonly do not resolve the magnetic structures: thus, the measurement of the polarization signal amplitude, which is always determined relative to the intensity, becomes more complicated because of spatial resolution, stray light, and thermodynamics of the solar atmosphere (Martínez González et al. 2006b). An accurate determination of magnetic field properties from the weak polarization signals prevalent in the quiet Sun in VIS lines is thus strongly dependent on the quality of the observations, the signal-to-noise ratio (Bellot Rubio \& Collados 2003; Rezaei et al. 2007), and the spatial resolution (Khomenko et al. 2005b). IR spectra at $1.56 \mu \mathrm{m}$ allow us to disentangle most of the ambiguities because the field strength can be inferred from the splitting of the polarization, or in some cases, even the intensity components of the spectral line for magnetic fields above around $400 \mathrm{G}$ (Beck 2006; Beck et al. 2007). It is thus unsurprising that studies before HINODE using solely VIS lines (Sánchez Almeida \& Lites 2000; Domínguez Cerdeña et al. 2003; Socas-Navarro \& Sánchez Almeida 2003; Lites \& Socas-Navarro 2004; Socas-Navarro \& Lites 2004) or IR lines (Lin 1995; Khomenko et al. 2003; 

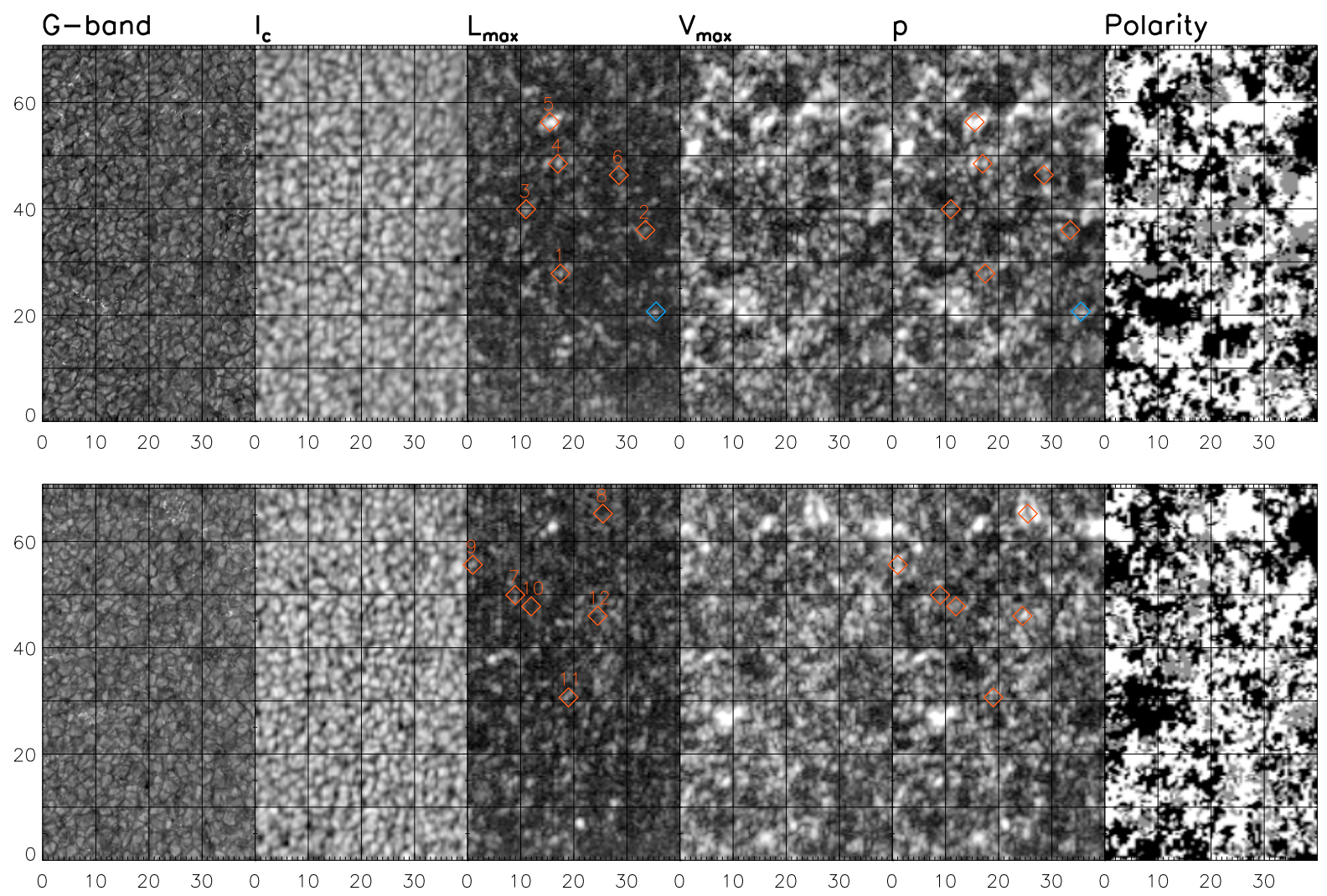

Fig. 1. Overview maps of the observations on 21.5.08. Top: TIP Op. 001, taken at around UT 8:30. Bottom: TIP Op. 005, from around UT 9:30. Left to right: G-band image, maps of $I_{\mathrm{c}}, L_{\max }, V_{\max }, p$, and polarity. Scale is in arcsec, grid lines have a 10 arcsec spacing. The blue diamonds denote the location of the profile shown in Fig. 4, the red diamonds the locations of those shown in Appendix A.

Martínez González et al. 2006a; Domínguez Cerdeña et al. 2006a) did not agree especially about the intrinsic field strength: the VIS observations indicated a significant fraction of strong $\mathrm{kG}$ fields, whereas the IR was dominated by weaker hG fields.

The contradictory findings from VIS or IR spectral lines could only recently be reconciled to some degree. Rezaei et al. (2007) found for the first time a predominance of weak fields in the internetwork (IN) from observations at $630 \mathrm{~nm}$ in a study of the relation between photospheric fields and chromospheric emission. They used ground-based observations obtained using the Kiepenheuer-Institute adaptive optics system (von der Lühe et al. 2003) and the POLIS spectropolarimeter (Beck et al. 2005b) at the German Vacuum Tower Telescope (VTT) in Izaña. Martínez González et al. (2008c) showed that for simultaneous observations in VIS and IR lines taken under identical seeing conditions, the VIS observations are compatible with the weak fields retrieved from the corresponding IR spectra. The VIS spectra alone were, however, found again to be biased towards $\mathrm{kG}$ fields because of the limitations of the weak-field limit.

The latest findings about the magnetic fields in the internetwork quiet Sun from the HINODE in VIS lines should thus again be compared and confirmed with corresponding observations in the IR. During an observation campaign in 2008, we were able to obtain IR spectra at $1.56 \mu \mathrm{m}$ in extremely good and stable seeing, yielding a nearly uniform spatial resolution in time and space close to the diffraction limit of the VTT at these wavelengths. We describe the observations in Sect. 2 and explain additional analysis steps and the inversion of the spectra with the SIR code in Sect. 3. The results are presented in Sect. 4 and summarized and discussed in Sect. 5. Our conclusions are given in Sect. 6.

\section{Observations}

On 2008 May 21, we observed a region of quiet Sun on disc centre with the Tenerife Infrared Polarimeter (TIP, Martínez Pillet et al. 1999; Collados et al. 2007) at the German Vacuum Tower Telescope (VTT) in Izaña. We used the $1.56 \mu \mathrm{m}$ range that includes two Zeeman-sensitive Fe I solar spectral lines at $1564.8 \mathrm{~nm}\left(g_{\text {eff }}=3\right)$ and $1565.2 \mathrm{~nm}\left(g_{\text {eff }}=1.5\right)$. The slit width of $100 \mu \mathrm{m}$ corresponded to $0 .{ }^{\prime} 45$. The slit was stepped in 0.5 steps across the solar image in the focal plane. The spatial sampling along the slit was 0 .' 175 ; the spectra were binned later to a 0.35 sampling, which is sufficient to sample the diffraction limit of the VTT $\left(0.0^{\prime} 6\right.$ at $\left.1.5 \mu \mathrm{m}\right)$. Because of the extremely good and stable seeing conditions, we used an integration time of $30 \mathrm{~s}$ per scan step. Two maps of $40^{\prime \prime} \times 70^{\prime \prime}$ were taken from UT 7:58 until 8:43 (Operation 001), and from UT 9:16 until 10:01 (Operation 005). The TIP data were reduced with the appropriate routines (e.g., Schlichenmaier \& Collados 2002) including the correction of the telescope polarization (Beck et al. 2005a). The noise level of the polarization signal in continuum windows is around $2 \times 10^{-4}$ of the continuum intensity.

We used a narrow-band G-band imaging channel for speckle-reconstructed context images and the TElecentric SOlar Spectrometer (TESOS, Kentischer et al. 1998) for G-band spectroscopy simultaneously with TIP. We aligned the specklereconstructed G-band images taken during the TIP maps with the same methods as described in Beck et al. (2007); the TESOS spectra were not considered in the present study.

Figure 1 shows overview maps of the observations: the aligned speckle-reconstructed G-band data, the continuum intensity $I_{\mathrm{c}}$ at around $1565 \mathrm{~nm}$, the maximum linear polarization 
signal $L_{\max }$ of the $1564.8 \mathrm{~nm}$ line, the maximum circular polarization signal $V_{\max }$, the polarization degree $p$, and finally, the polarity of the magnetic fields that indicates whether the field lines were (anti)parallel to the line of sight (LOS). The polarity was defined as \pm 1 (black and white in Fig. 1) from the order of minimum and maximum Stokes $V$ signal around the $1564.8 \mathrm{~nm}$ line core for all locations with a polarization degree above the threshold discussed below. Locations without significant polarization signal appear grey in the polarity map. We defined $L_{\max }, V_{\max }$, and $p$ as the strongest polarization signal in a small wavelength range around the line core of $1564.8 \mathrm{~nm}$ in $L=\sqrt{Q^{2}+U^{2}}$, Stokes $V$, and $P=\sqrt{Q^{2}+U^{2}+V^{2}}$, respectively. Assuming that the speckle-reconstructed G-band data (leftmost image) is a close representation of the true solar surface structure, the TIP data exhibit the same structure only degraded by the limited spatial sampling, despite the $30 \mathrm{~s}$ integration time. The cadence of the G-band data was interrupted in both observations close to the end of scanning, and the G-band data has no exact timing information. It was thus impossible to obtain a good alignment with $I_{\mathrm{c}}$ for the last approximately $10^{\prime \prime}$ of the scans. The maps of $L_{\max }, V_{\max }$, and $p$ are all displayed with an identical upper threshold in polarization amplitude of $1 \%$ of $I_{\mathrm{c}}$. The maps of $p$ are dominated by the contribution from circular polarization, i.e., nearly vertical magnetic fields, but there are also several locations with strong linear polarization signal. We note that only a small fraction of the magnetic fields leads to bright points in the G-band (e.g., $x / y=8-15 / 25-20^{\prime \prime}$ in the upper left image), as also found by de Wijn et al. (2008). The second map (lower row), taken around an hour later, again shows the strongest and stable magnetic flux concentrations of the previous observation displaced by around $5^{\prime \prime}$ : the black patch in the polarity map at $x / y=38 / 55^{\prime \prime}$, or the small white patch at $18 / 58^{\prime \prime}$, can be reidentified in the 2nd map as well at 38/65" and 18/63", respectively.

\section{Data analysis}

To compare with the study of LI08, we also calculated the total linear and circular polarization, $L_{\mathrm{tot}}$ and $V_{\mathrm{tot}}$, by integrating the absolute polarization signal $L(\lambda)$ and $|V(\lambda)|$ with wavelength. These quantities are related to the longitudinal $\left(B_{\mathrm{L}}\right)$ and transversal $\left(B_{\mathrm{T}}\right)$ component of the magnetic field. The (anti)parallel direction of the longitudinal fields is introduced into $V_{\text {tot }}$ again by a multiplication with the polarity map. Figure 2 displays the maps of $L_{\mathrm{tot}}$ and $V_{\mathrm{tot}}$. We show in addition maps of the ratio of linear to circular polarization, $L / V$. The ratio was calculated for an average of a few spectral pixels $(\sim 10 \mathrm{pm})$ near the location of the highest $V$ signal of $1564.8 \mathrm{~nm}$ (see Beck 2006). The spatial pattern in these maps is strikingly similar to that found by LI08: the polarization signal shows "voids" of some granules extent (about $10^{\prime \prime}$ diameter in $L_{\mathrm{tot}}, 3-5^{\prime \prime}$ in $\left.V_{\mathrm{tot}}\right)$ that are free of any clear signal; the linear polarization signal appears in the shape of small "blobs" around the edges of the voids. The blobs of enhanced $L_{\text {tot }}$ are slightly smaller than individual granules (cf. Ishikawa et al. 2008). In the ratio $L / V$ (right column), several more locations with stronger linear than circular polarization show up in the form of similar blobs as in $L_{\mathrm{tot}}$. For example, at $x / y=26 / 26^{\prime \prime}$ and 30/30" in the upper left image, two blobs appear in $L_{\text {tot }}$ (blue rectangles), whereas in $L / V$ (upper right) a third blob is visible between them at $x / y=28 / 28^{\prime \prime}$.

Figure 3 shows one example of slit spectra from each observation. There is a residual (vertical) fringe pattern in $Q U V$ of Op. 001, which originates from imperfect flatfielding, of maximum amplitude around 0.0005 of $I_{\mathrm{c}}$ in Stokes $U$, and smaller
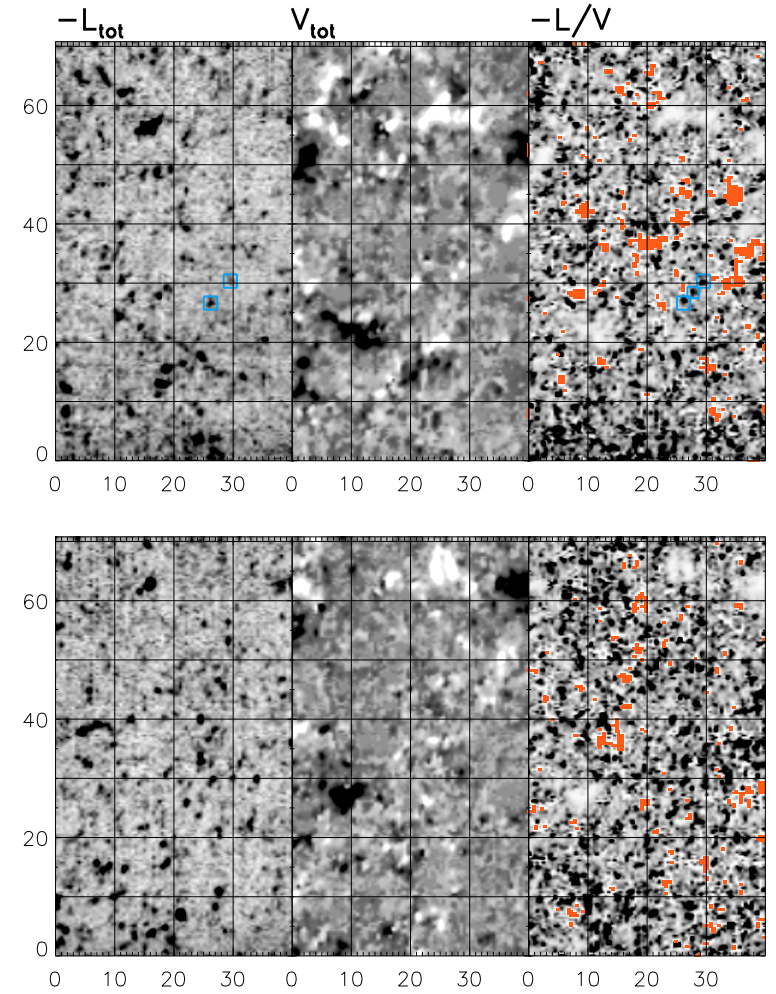

Fig. 2. Left to right: $L_{\mathrm{tot}}, V_{\mathrm{tot}}$ times polarity, ratio $L / V$. The red patches in $L / V$ denote locations without any clear $V$ signal to divide with. First and last map are displayed inverted. The blue rectangles in the upper left image denote two patches of large $L_{\mathrm{tot}}$, between which in $L / V$ (upper right image) another one shows up. Top/bottom: TIP Op. 001 and Op. 005. Scale is in arcsec, grid lines have a 10 arcsec spacing.

amplitude in $Q$ and $V$. The amplitude of the fringes is so small that they are not visible, even in the wavelength-integrated map of $L_{\text {tot }}$ (see Fig. 2). The fringes are presumably caused by the glass window that protects the CCD chip inside the cryostat. We applied some additional corrections to the lower part of the slit from 0 " to around $20^{\prime \prime}$, but emphasize that it could be improved by applying a PCA analysis as in Martínez González et al. (2008c,a). For the 2nd observation, residual fringes are much weaker and can only be seen in Stokes $Q$. The inversion code is unable to reproduce the pattern because of the combination of its limited number of degrees of freedom, the least squares minimization that it uses, and the extent of the fringes in wavelength. The characteristic wavelength of the fringe pattern is about twice as wide as one of the solar spectral lines; with the chosen setup, the inversion code has no degrees of freedom (see the next paragraph) to produce such a pattern. In this case, two synthetic profiles will show small deviations from the observed profile: the one that includes the fringe pattern (which the inversion code cannot generate), and the one that ignores the additional modulation. We thus assume that the influence of the fringes on the results is negligible. Comparing the two spectral lines at $1564.8 \mathrm{~nm}$ and $1565.2 \mathrm{~nm}$, it is clear that most of the linear polarization signals seen in the more sensitive $1564.8 \mathrm{~nm}$ line are absent in the other, as already noted by Khomenko et al. (2003); only the strongest linear polarization signals appear in both lines. Almost every profile along the slit exhibits a significant circular polarization signal in both spectra.

The noise level in the spectra is low enough for us to run a "standard" inversion of the spectra with the SIR code 


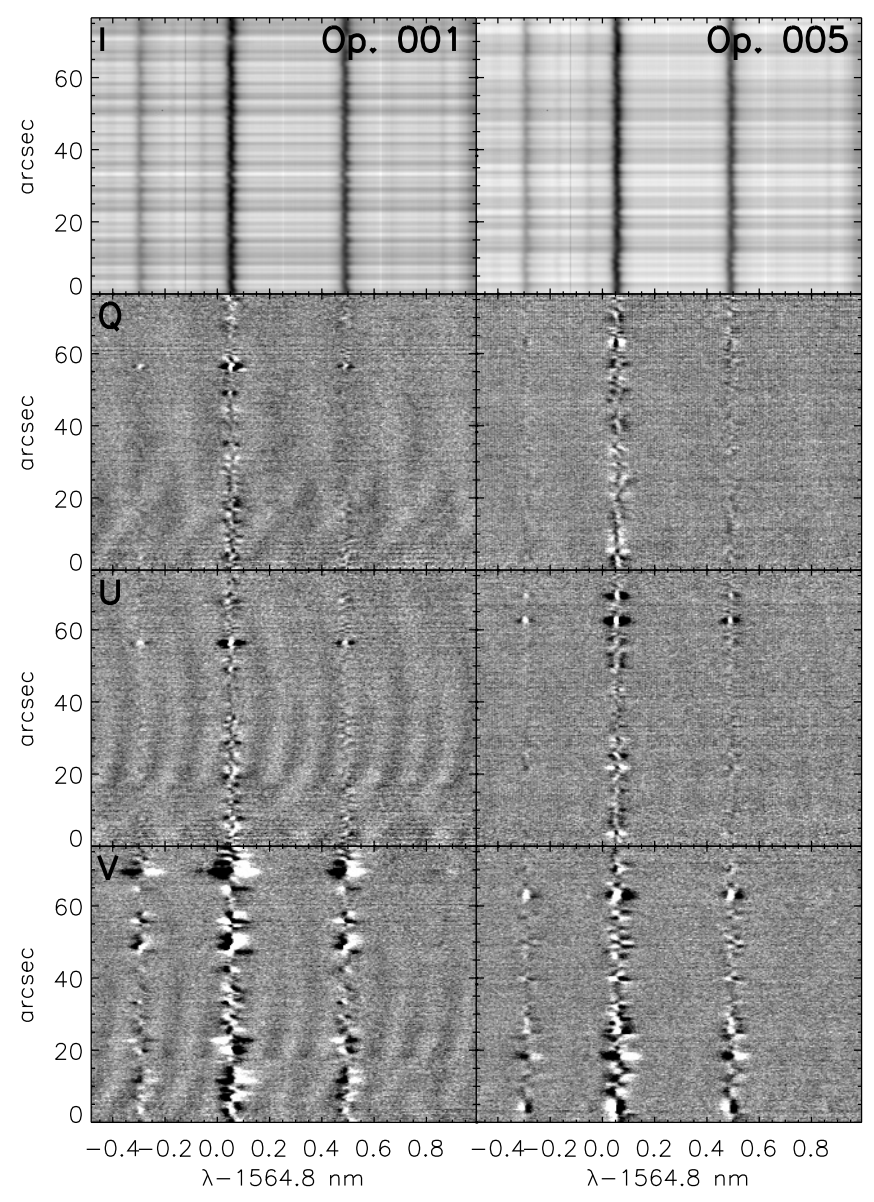

Fig. 3. Example of top to bottom IQUV spectra for one scan step from Op. 001 at left and Op. 005 at right. The display threshold for $Q U V$ was set to $\pm 0.001 I_{\mathrm{c}}$.

(Ruiz Cobo \& del Toro Iniesta 1992). "Standard" means that we do not modify the inversion setup significantly to optimize it for the special type of data, to get a hopefully unbiased first guess of the magnetic field properties without enforcing a priori knowledge. We used the same setup as Beck et al. (2007): in each pixel, we assumed one field-free and one magnetic atmosphere component, with additional stray light. We only modified the inversion to use three nodes in temperature for the magnetic component, and place equal weight on the polarization components $Q, U$, and $V$. The parameters of the magnetic field and the LOS velocities were assumed to be constant with optical depth. The 2-C+stray light setup mimics observations of unresolved magnetic flux (magnetic component) in equally unresolved intergranular lanes (field-free component), whereas the stray light corresponds to the granular contribution to the spectra $^{1}$. Figures 4, A.1 and A.2 show several examples of observed spectra together with the best-fit spectra retrieved by the inversion. We selected locations with a low polarization degree and mostly higher linear than circular polarization signal for the figures. For all of these examples, the signal in the less sensitive $1565.2 \mathrm{~nm}$ line is already close to or below the noise level, whereas the $1564.8 \mathrm{~nm}$ line still yields a "significant" signal that the inversion code can reproduce.

In defining "significant", we used the polarization degree $p$. The routine that performed the inversion used a threshold of

1 The average spectrum used as stray-light contribution is blue-shifted and has a continuum intensity of unity.
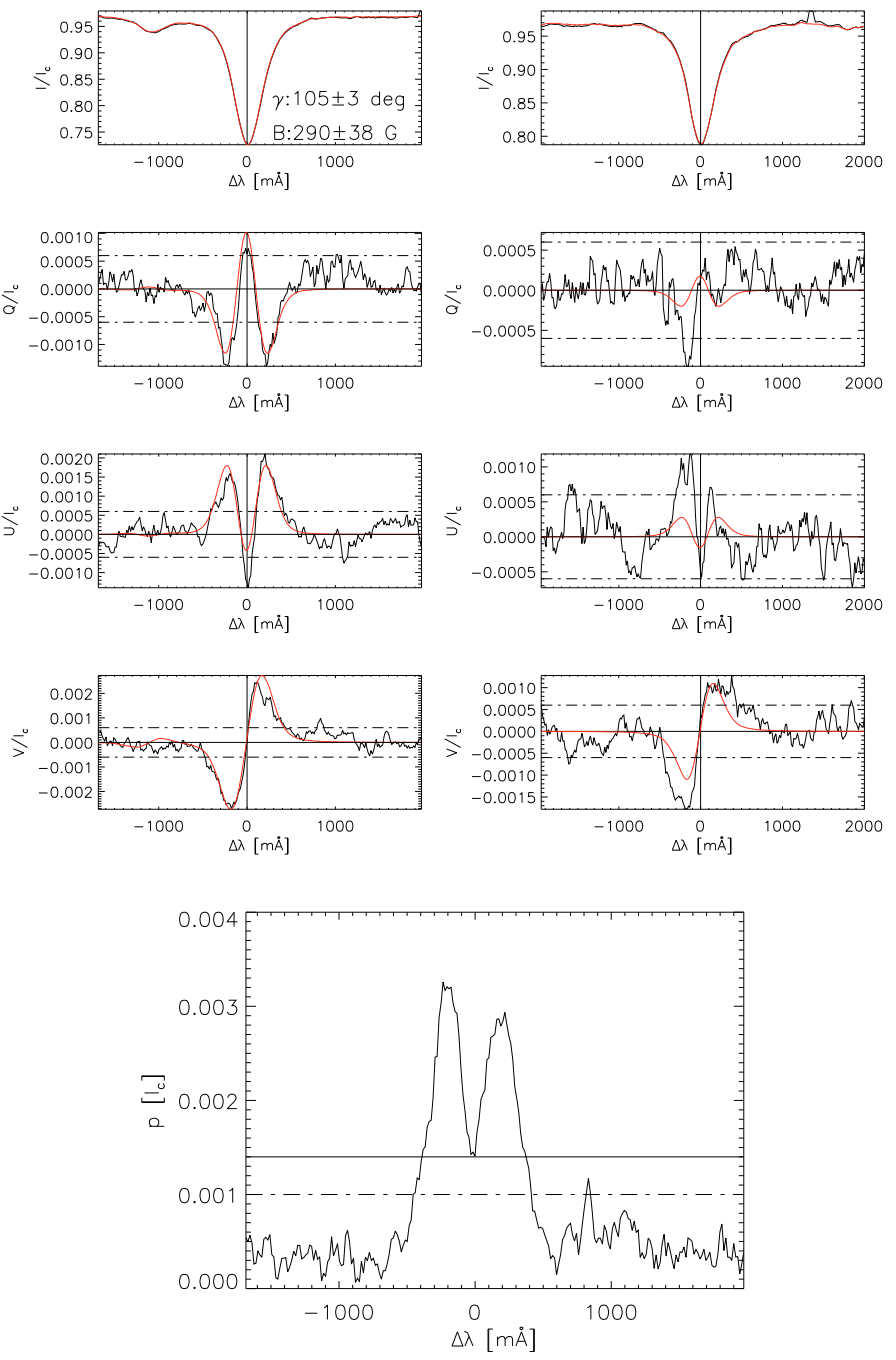

Fig. 4. Top: example of inverted spectra of the $1564.8 \mathrm{~nm}$ (left column) and $1565.2 \mathrm{~nm}$ line (right column). Black lines: observed spectra of top to bottom IQUV; red: best-fit profile of the inversion. The dashdotted horizontal lines mark three times the rms noise level, the solid horizontal line the zero level. The vertical solid line denotes the rest wavelength. Bottom: polarization degree of the $1564.8 \mathrm{~nm}$ line with the threshold for inversion (dash-dotted) and final rejection (horizontal solid line).

$1 \times 10^{-3}$ of $I_{\mathrm{c}}$ : if the maximal value of $p$ of either $1564.8 \mathrm{~nm}$ or $1565.2 \mathrm{~nm}$ was above this limit, the 2 -C inversion with a magnetic component was executed. As can be seen in Figs. 4 or A.3 (right column, 3rd row), this level is reached already by (most probably) noise alone in two out of 13 cases for $1564.8 \mathrm{~nm}$. We thus have set a slightly higher threshold for the analysis of the inversion result and rejected all profiles with $p<1.4 \times 10^{-3}$ of $I_{\mathrm{c}}$ in $1564.8 \mathrm{~nm}$. In both maps, around $63 \%$ of the pixels are above the threshold corresponding to 4 times the noise rms in $p$ $\left(3.4 \times 10^{-4}\right.$ of $\left.I_{\mathrm{c}}\right)$.

Sheminova (2009) found that about $70 \%$ of the surface area in a 2-D MHD simulation was covered by magnetic fields. OR07 measured a fraction of $630 \mathrm{~nm}$ profiles of $87 \%$ and $35.5 \%$ that had a polarization degree of 3 and 4.5 times the noise level of about $1 \times 10^{-3}$ in their HINODE data. These numbers thus all agree that the area fraction covered by magnetic fields or exhibiting polarization signal should be above $50 \%$, but Fig. 5 infers that the fraction of profiles with $p>p_{\text {thresh strongly de- }}$ pends on the threshold used. For comparison with a theoretical 


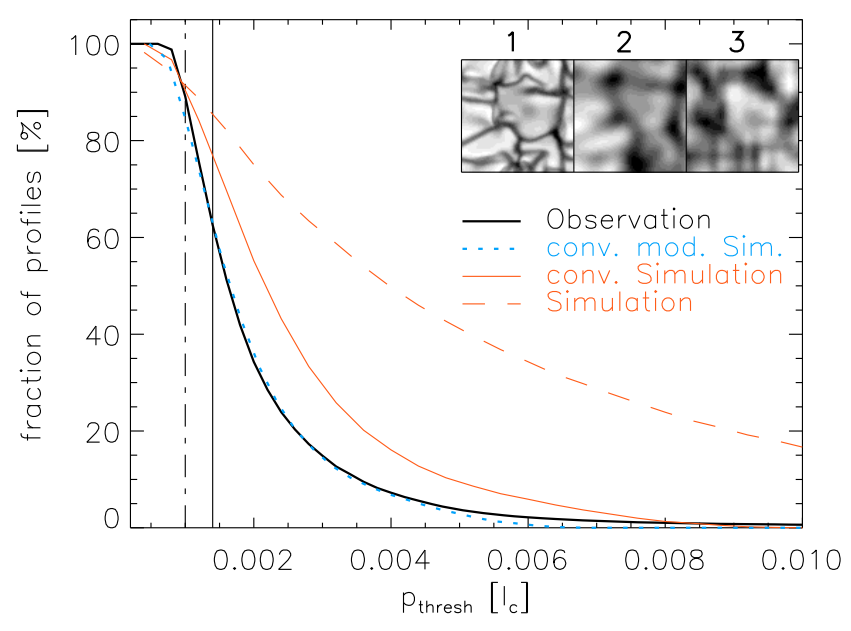

Fig. 5. Fraction of profiles with $p>p_{\text {thresh. Dash-dotted and solid ver- }}$ tical lines denote the inversion and final rejection threshold in the observations, respectively. The inlets show continuum intensity maps of the original (1) and convolved simulation data (2), and a section of the same size from a TIP map (3).

calculation, we derived the same fraction of profiles with $p>$ $p_{\text {thresh }}$ from spectra of $1564.8 \mathrm{~nm}$ synthesized from a simulation run with the $\mathrm{Co}^{5}$ bold code described in Schaffenberger et al. (2005, 2006) and previously used in Steiner et al. (2008). The snapshot was taken from the h20 run with an initial homogeneous horizontal magnetic field of $20 \mathrm{G}$. The simulation box corresponded to a 6.' 6 square; its spatial resolution is much higher than for the present observations (compare the insets 1 and 3). We thus convolved the simulations' spectra spatially with a Gaussian kernel of FWHM $\sim 0.9$. We also added a noise level of $2.2 \times 10^{-4}$ to them. The original simulation spectra without convolution and noise (red dashed in Fig. 5) contain a much higher fraction of profiles with a high polarization degree than the observations. After the convolution, the curve already roughly matches that of the observations (red solid). We ascribe the remaining difference to the imperfect match of the spatial resolution, the different spatial sampling, and the much larger solar area studied by the observations $\left(40^{\prime \prime} \times 70^{\prime \prime}\right)$. We note only that the convolved simulated spectra can be matched almost perfectly to the observations by compressing the curve in the abscissa by a factor of 1.5 (blue dotted line). This compression can be understood as a reduction in the polarization degree that could be due to, e.g., the stray light that was not included in the convolution, or the usage of a Gaussian kernel with its steep drop with distance. A reduction by 1.5 may sound large, but one has to consider that the effect is to change, e.g., a $0.6 \%$ polarization level to instead $0.4 \%$, for which only a small amount of additional stray light is needed. The slopes of all curves clearly change when the noise level is reached; the fraction of profiles is constantly close to $100 \%$ for thresholds below the noise. We suggest that the fraction of profiles above a polarization threshold can be easily used to match the spatial resolution of simulation spectra to observations, and is also a good indicator of the spatial resolution achieved in a specific observation.

\section{Inversion results}

The inversion code constructs its synthetic spectra, $S$, by the following combination of inversion components:

$S=\alpha \cdot S_{\text {stray }}+(1-\alpha) \cdot\left(\beta \cdot S_{\text {magnetic }}+(1-\beta) \cdot S_{\text {field-free }}\right)$,

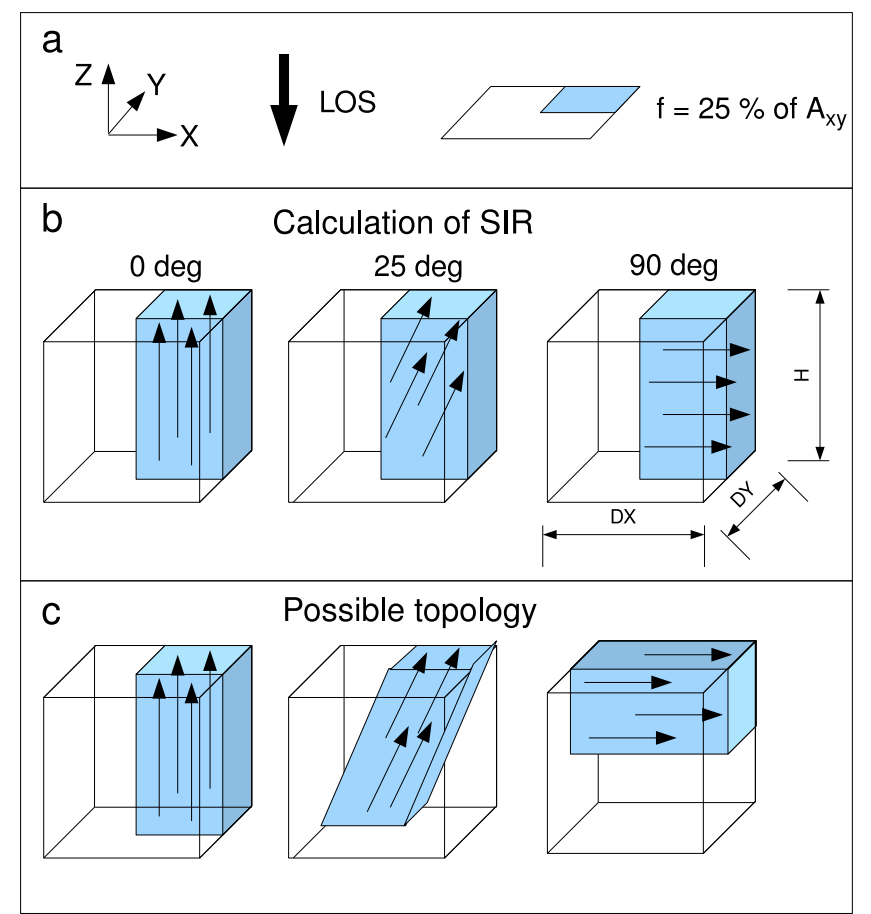

Fig. 6. Comparison of the geometry in the calculation of SIR and a possible topology inside the resolution element. a) Left to right: reference frame, LOS direction, and filling fraction; b) geometry in the calculation by SIR for field inclinations to the LOS of left to right 0,25 , and 90 degrees; c) possible solution for the real topology.

where $S_{\text {stray }}$ is the average profile of the complete map that is taken to describe the stray-light contribution and $\alpha$ its fractional contribution to the spectrum. The relative contributions of the magnetic and the field-free component are given by $\beta$ and $1-\beta$, respectively. Assuming that $S_{\text {stray }}$ corresponds to another fieldfree component of a fixed velocity, we define the filling fraction $f$ of the magnetic fields by $f=(1-\alpha) \cdot \beta$. The additional quantities from the inversion code are the field strength, $B$, field inclination to the LOS, $\gamma$, field azimuth, $\psi$, LOS velocities for each component, and the temperature stratification. The observations were on disc centre, thus the LOS inclination $\gamma$ is identical to the field inclination to the surface normal.

From the inversion results, we estimated the magnetic flux by

$\Phi=f \cdot B \cdot A$,

where $A$ is the area corresponding to a single pixel of $0.5 \times$ 0.35 .

For this estimate of the magnetic flux, we did not consider the field inclination. We justify this by assuming that the volume contributing to the spectra from one pixel corresponds roughly to $\mathrm{a}(275 \mathrm{~km})^{3}$ cube. The spatial extent in the horizontal dimensions $(\mathrm{d} x, \mathrm{~d} y)$ is $360 \mathrm{~km}$ by $250 \mathrm{~km}$. For the extension of the formation height, $H$, of the IR line at $1564.8 \mathrm{~nm}$, Cabrera Solana et al. (2005) give a range of around two dex in $\log \tau$, e.g., from around 0 to -2 for velocity perturbations or from 0.5 to -2.5 for magnetic fields. In the HSRA model atmosphere (Gingerich et al. 1971) that they used, this range corresponds to around $250 \mathrm{~km}$. Figure 6 shows one possible way how the calculation of SIR, which determines a filling fraction in the horizontal plane and then solves the radiative transfer along the LOS, could be related to the real topology inside the pixel. We assumed a constant filling fraction of $25 \%$ of the horizontal area $A_{x y}$, and plotted three cases for field inclinations to the LOS of 0,25 , and 90 degrees. To obtain the magnetic flux, the filling fraction has to be 


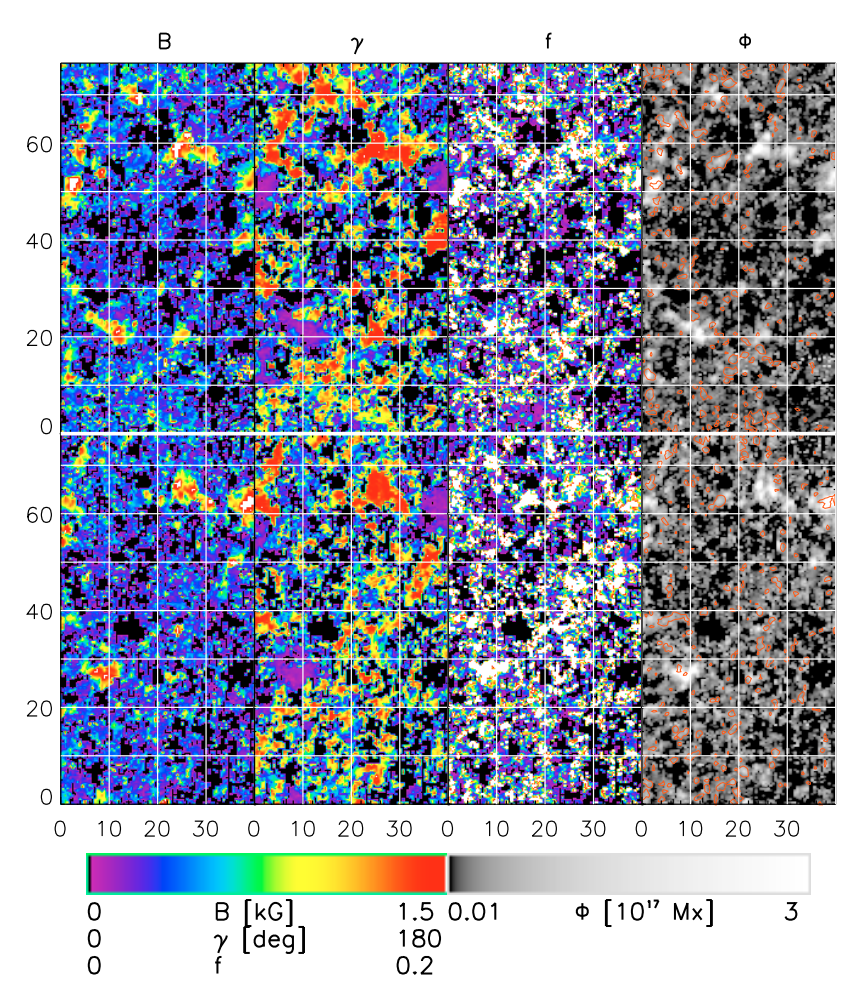

Fig. 7. Inversion results of Op. 001 (top row) and Op. 005 (bottom row). Left to right: field strength, LOS inclination, filling fraction, total flux. Red contours in $\Phi$ trace strong linear polarization signals. The display ranges are given by the labels of the color bar at the bottom. Pixels not inverted are shown black.

multiplied by the corresponding area perpendicular to the field direction. For horizontal magnetic fields, the respective area is, however, not $A_{x y}$ but $A_{x z}$ or $A_{y z}$, depending on the field azimuth. If $\mathrm{d} x \sim \mathrm{d} y \sim H$, the surfaces of the cube are equal and thus the field orientation can be ignored to first order. This is only an approximation, since for example in the 90-deg-case, SIR calculates the synthetic profile assuming that the horizontal fields are present everywhere inside the formation height, which does not necessarily provide the same result as when they fill only half the formation height with a double filling fraction.

Figure 7 shows maps of $B, \gamma, f$, and $\Phi$ for the two observations. Magnetic fields with $\mathrm{kG}$ or more are restricted to vertical fields ( 1 st and 2 nd column, $\gamma \sim 0$ or $180^{\circ}$ ). The $\mathrm{kG}$ fields show a high magnetic filling fraction, but parts with horizontal fields can also reach the same level in $f$. The more inclined fields, however, do not appear prominently in the flux map (rightmost column). We overplotted contour lines tracing the strongest linear polarization signal (cf. Fig. 2) onto the flux map. Locations of high magnetic flux are almost exclusively related to vertical fields with little linear polarization signal.

The total amount of magnetic flux in both maps calculated with Eq. (2) is around $6 \times 10^{20} \mathrm{Mx}$. There is a slight imbalance of $53 \%$ to $47 \%$ for magnetic flux parallel or antiparallel to the LOS. Including the field inclination in the calculation to obtain the LOS magnetic flux by multiplying $\Phi$ with $\cos \gamma$, the imbalance is exactly reversed. Since the fraction is close to $50 \%$, we conclude that the FOV provides a sufficiently large statistical sample of quiet Sun regions where no strong bias to a particular field orientation is expected. The histogram of inclinations using the full range from 0 to $180 \mathrm{deg}$ was also fairly symmetric, so in the following we have converted the field inclinations above
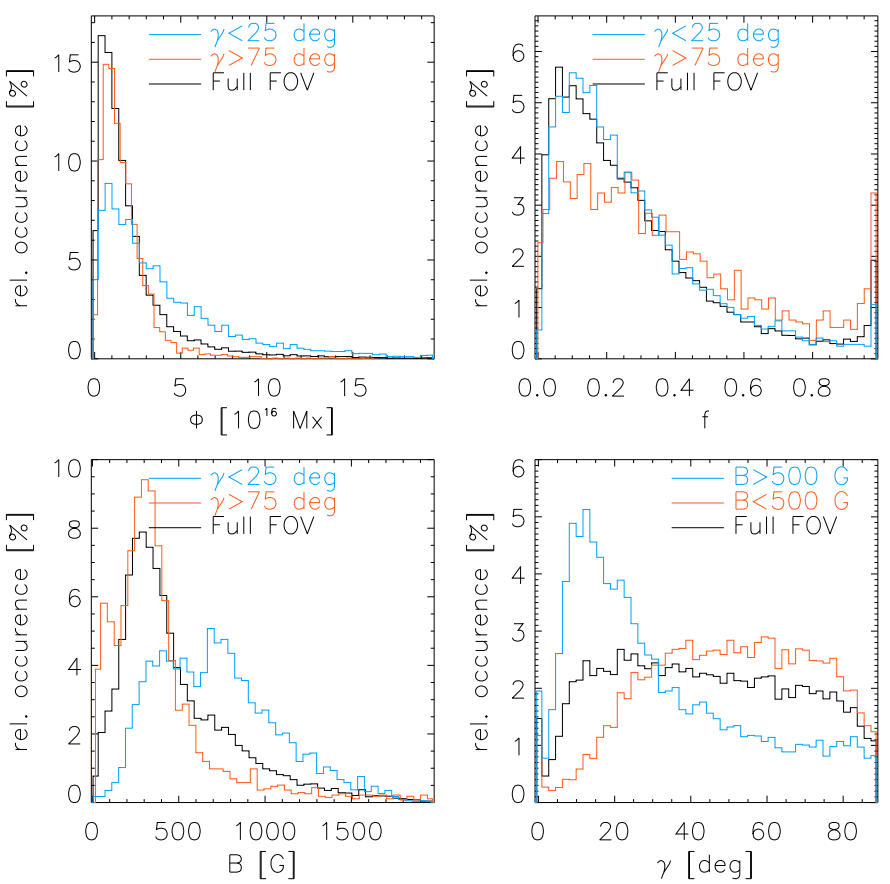

Fig. 8. Histograms of (clockwise, starting left top) magnetic flux, filling fraction, field inclination, field strength. Blue and red lines correspond to the least/most inclined fields, black lines to the full FOV.

90 degrees to their corresponding values in the range from 0 to 90 degrees.

Figure 8 shows histograms of $B, \Phi, \gamma$, and $f$. We calculated the histograms for three different samples: the full FOV (black), all locations with nearly vertical magnetic fields (blue, $\gamma<25^{\circ}$ ), and nearly horizontal magnetic fields (red, $\gamma>75^{\circ}$ ). For the histogram of $\gamma$, we separated the data points into those for magnetic fields above and below $500 \mathrm{G}$. The visual impression of the 2-D maps is confirmed by the histograms: the nearly vertical fields correspond to the locations of large magnetic flux and high field strength (300-1000 G); the nearly horizontal fields are limited to field strengths below around $500 \mathrm{G}$.

The distribution of magnetic field strength for the full FOV agrees with previous observations of the IR lines (Khomenko et al. 2003; Martínez González et al. 2008c) or the field strength distribution derived by Sheminova (2009) from 2-D MHD simulations, especially for the most probable field strength of around $250 \mathrm{G}$. We emphasize that a) the "bump" at around $1.5 \mathrm{kG}$ commonly found when analyzing $630 \mathrm{~nm}$ data (e.g., Domínguez Cerdeña et al. 2006a, OR07) is missing, even when the network locations inside the FOV are not masked out, and that b) the decrease in the relative occurrence of fields below $250 \mathrm{G}$ is not due to the polarization detection limit. Figure A.2 shows 3 examples of weak fields below $200 \mathrm{G}$ (2nd row) that could still be reliably reproduced by the inversion. The spatial resolution of observations is also crucial to the detection of these weak fields (see Fig. 5). The distribution of the nearly horizontal fields (red curve at bottom left of Fig. 8) shows an enhanced fraction of these weak fields below $200 \mathrm{G}$, which implies that the detection limit due to the noise is not yet reached at $200 \mathrm{G}$. In the question of the seemingly preference for $\mathrm{kG}$ fields in the analysis of $630 \mathrm{~nm}$ data, Martínez González et al. (2006b) cautioned that the thermodynamics must be treated consistently in an analysis of the $630 \mathrm{~nm}$ line pair in the weak field limit. Rezaei et al. (2007) were able to derive a distribution without the bump using a SIR inversion of $630 \mathrm{~nm}$ data of lower spatial 

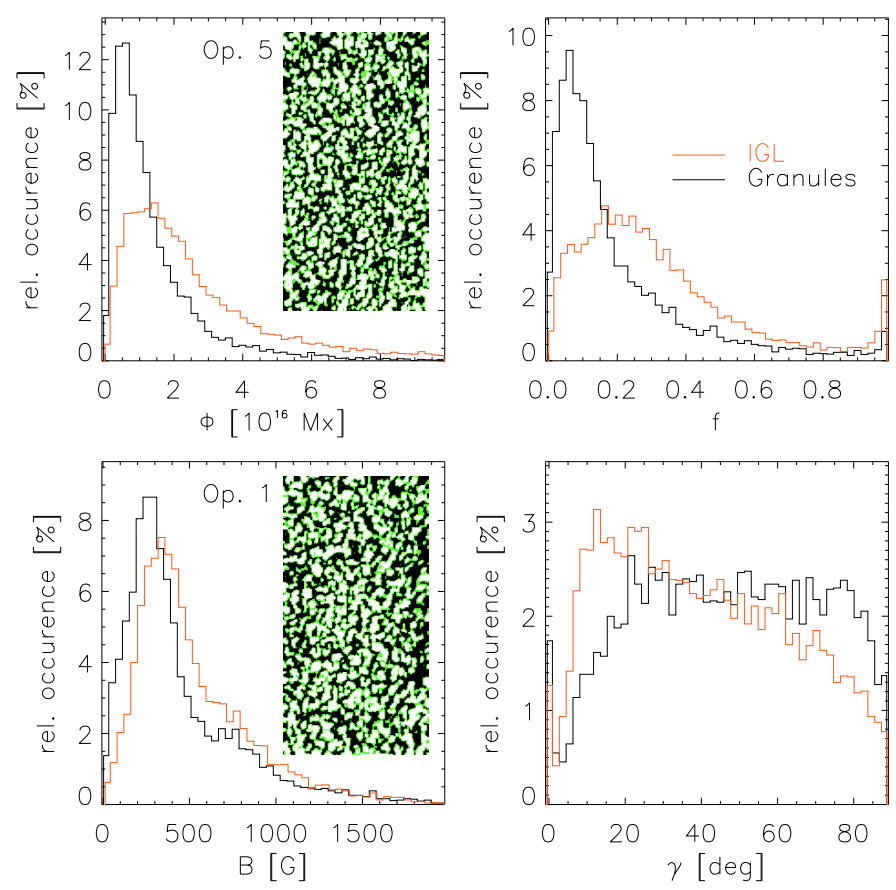

Fig. 9. The same histograms as in Fig. 8 but separated into granules (black lines) and intergranular lanes (red). The inlets show the masks used for the separation.

resolution than available nowadays in HINODE observations. It would be worthwhile to analyse the HINODE data without employing the Milne-Eddington (ME) approximation to determine whether the field strength distribution changes with a more sophisticated treatment of the thermodynamics.

The field inclination shows some clear trends: for fields below $500 \mathrm{G}$, an inclination above $45^{\circ}$ is preferred with a plateau of constant probability up to $90^{\circ}$, whereas strong fields above $500 \mathrm{G}$ are restricted to an inclination of $35^{\circ}$ or less. The distribution for the full FOV shows a small peak near $0^{\circ}$ inclination (三the very small area fraction of "network" fields inside the FOV), and then a rapid increase in occurrence up to $15^{\circ}$ followed by a slow reduction towards $90^{\circ}$. The shape of the distribution matches more closely the one given by OR07 in their Fig. 3 for the MHD simulation run of Vögler et al. (2005), than the one that these authors derived from their observations.

A second method of classification into different type of structures is to separate the FOV into granules and intergranular lanes (IGLs). We derived a mask using the continuum intensity maps and a threshold of 0.995 and 1.005 of $I_{\mathrm{c}}$ to define IGLs (black in the inlets in Fig. 9) and granules (white), respectively. Locations with an intensity between the two values were excluded (green). The masks retain the granulation pattern of the continuum intensity. At the near-infrared wavelength of the observations and at their spatial sampling, magnetic elements are not bright in the continuum at the disc centre, and thus should not have unintentionally been counted as part of the granular sample. We then calculated the histograms of the same quantities as in Fig. 8. The pattern is very similar to that in Fig. 8: in the IGLs, one has a predominance of more vertical, stronger fields of higher magnetic flux. A strong difference between granules and IGLs is seen in the magnetic filling fraction. The IGLs contain a larger contribution of high filling fractions centred on $0.2-0.3$, whereas the granules have a distribution that peaks close to $f=0$ and drops steeply for larger $f$. This indicates that the magnetic flux in (or above) granules is more diffuse than in the IGLs and becomes more dispersed than concentrated, whereas in the IGLs the converging granular flows advect and concentrate magnetic flux.

The relative amount of magnetic flux in concentrated vertical fields in IGLs and the diffuse weak horizontal fields in granules is of strong interest, e.g., for reconciling Hanle and Zeeman measurements at low spatial resolution, which have yielded quite different average magnetic flux densities, Zeeman-based observations being lower by around an order of magnitude. The histograms of Figs. 8 and 9 indicate a tight relation between field strength, magnetic flux, and field inclination. We thus also created scatter plots of inclination versus the other two quantities (top two panels of Fig. 10). We remark that the field inclination did not enter into the calculation of the magnetic flux, but only the filling fraction and field strength. In the scatter plots, there is a clear trend for the inclined fields above $45^{\circ}$ to be limited to below $500 \mathrm{G}$ and below around $5 \times 10^{16} \mathrm{Mx}$. Higher fluxes are only achieved by nearly vertical fields (see Ishikawa \& Tsuneta 2009, their Fig. 6). To obtain the total amount of magnetic flux as a function of inclination, we added the magnetic fluxes at all locations with a field inclination in $\left[\gamma, \gamma+1^{\circ}\right]$ (lower panel). The distribution is similar to the scatter plot at top of Fig. 10; we find that around $2 / 3$ of the total magnetic flux comes from magnetic fields with $\gamma<45^{\circ}$.

In the observations of low spatial resolution, the weaker and more inclined magnetic fields could not be detected because their linear polarization signal had been spatially smoothed to values below the noise level (see Fig. 11, observations are described in detail in Khomenko et al. 2005a; Martínez González et al. 2008c). These data of 2003 were acquired for the same spectral range as the present investigation and an identical integration time of $30 \mathrm{~s}$ per spectrum, but an image correction of only a correlation tracker was used to remove image shifts during the exposure. A single position at $\left(48^{\prime \prime}, 22^{\prime \prime}\right)$ in a $60^{\prime \prime} \times 35^{\prime \prime}$ FOV shows significant linear polarization signal that would indicate inclined magnetic fields. Khomenko et al. (2003), however, were able to detect linear polarization signals in 50-s integration data from TIP taken on 2000 July 29 and 2000 September $5^{2}$. They found that around $20 \%$ of the pixels inside the FOV exhibited a significant linear polarization signal that could be analysed quantitatively.

\section{Discussion}

\subsection{General properties of the QS magnetic fields}

The weak horizontal magnetic field of the solar internetwork regions is only detectable with observations of high spatial resolution and high polarimetric sensitivity. The presence of these fields was expected from Hanle measurements, and evidence of them has been found in some observations before (Lites et al. 1996; Khomenko et al. 2003; Harvey et al. 2007; Martínez González et al. 2008b,c). They have only been seen prominently with data from the spectropolarimeter onboard the HINODE satellite (Kosugi et al. 2007), as reported by OR07 and LI08. By comparing the present Fig. 2 with Figs. 2 and 7 of LI08, we conclude that the present observations trace the same solar structures, i.e., ground-based observations aided by real-time adaptive optics correction can reach the required spatial resolution during spells of excellent seeing conditions, and hence, the results of the two independent sets of data should be compatible.

Some of the present findings disagree with those of the HINODE data. However, we propose that the disagreements

\footnotetext{
2 See http://www3.kis. uni-freiburg. de/ cbeck/ TIP_archive/TIP_archivemain.html
} 

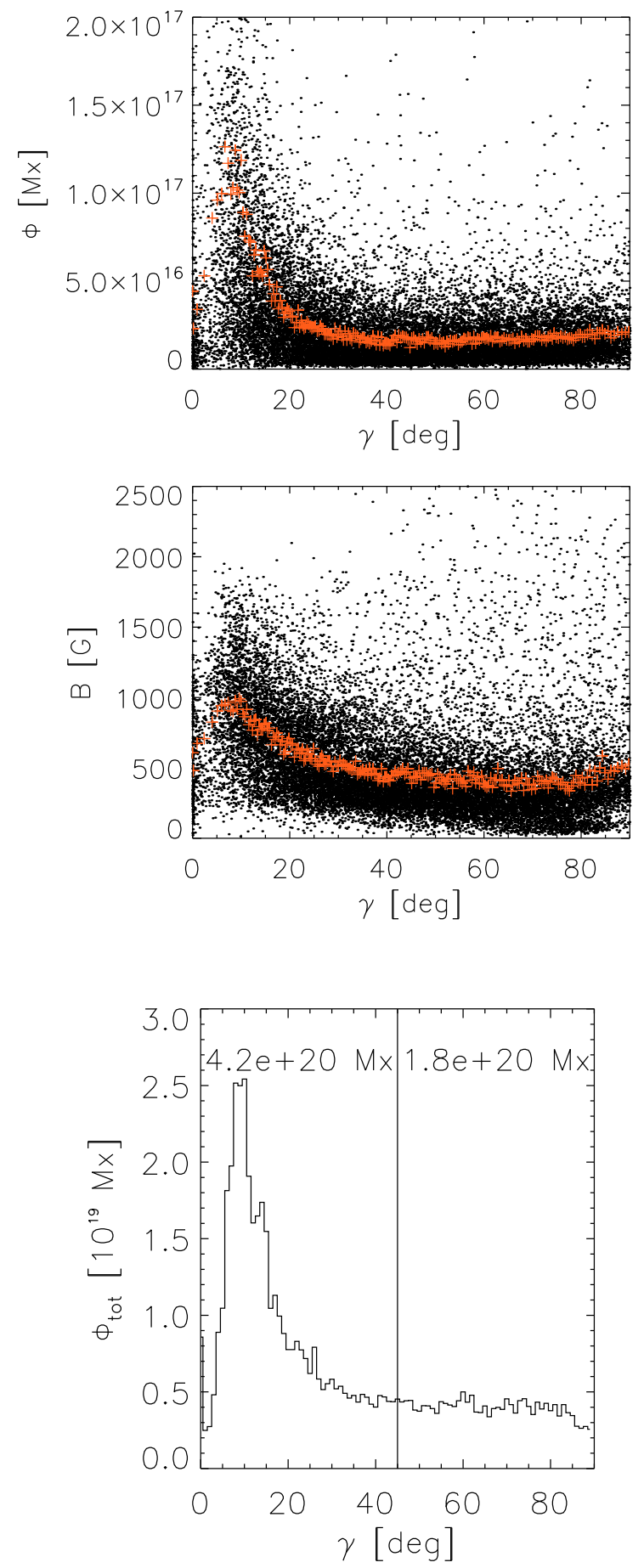

Fig. 10. Scatter plots of magnetic flux versus inclination (top) and field strength versus inclination (middle). Red crosses are binned data. Bottom: total magnetic flux in the full FOV as function of field inclination in 1 deg bins. The black vertical line marks $45^{\circ}$; the numbers give the total magnetic flux of all fields below or above this limit.

are mainly caused by the analysis methods, the different magnetic sensitivities of the spectral lines, and a physical reason. The distributions of field strength again exhibit the mismatch between IR and VIS found in previous studies, i.e., a significant fraction of $\mathrm{kG}$ fields in VIS (OR07) that does not appear for IR data. Bellot Rubio \& Collados (2003), Martínez González et al. (2006b), and Rezaei et al. (2007) demonstrated that this feature is related to the noise level of the data and the

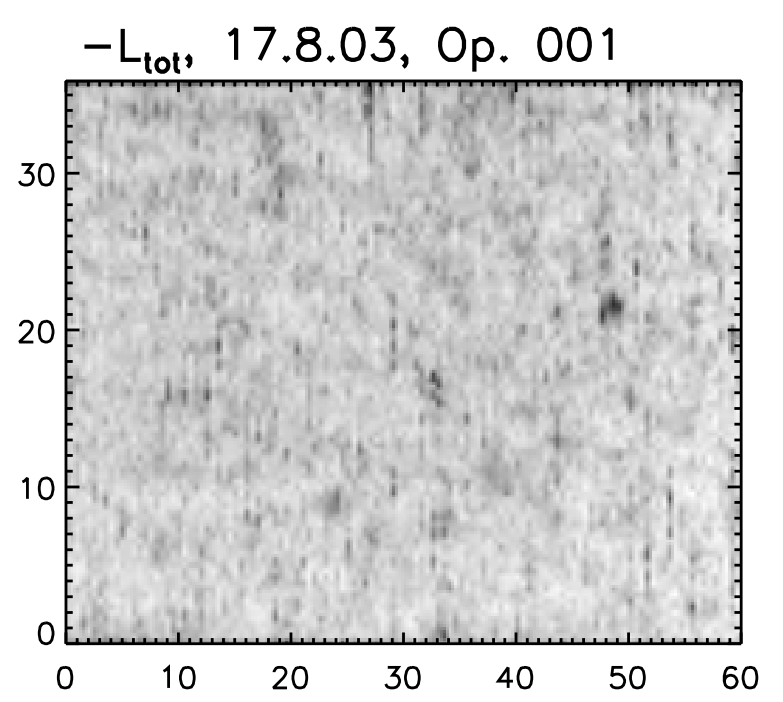

Fig. 11. Linear polarization map $L_{\text {tot }}$ of an observation of quiet Sun with TIP@1.56 micron from 17.8.2003. Tick marks are in arcsec.

thermodynamics, because IR and VIS spectra together can be reproduced simultaneously by weak fields (Martínez González et al. 2008c). The usage of a ME method in OR07 possibly also contributes to this effect. The field strength distribution here peaks at around $250 \mathrm{G}$, whereas Orozco Suárez et al. (2007) give $90 \mathrm{G}$. This may again be related to the weak-field limit of the $630 \mathrm{~nm}$ lines and the ME approximation, which makes the VIS lines less reliable than the stronger splitting IR lines. The magnetic filling fraction $f$ compares fairly well to one minus the stray light contribution of OR07; magnetic filling fractions peak at around 0.1 here and at 0.2 in OR07. The field inclinations differ the most: we do not find any increase in the distribution for horizontal fields, but instead a probability that declines slowly with inclination for fields with inclinations above $20^{\circ}$. OR07 and LI 08 both found a clear dominance of horizontal over vertical fields and transversal over longitudinal flux. With the same caveat as in LI08 that the vertical and horizontal extent of the transverse fields cannot be derived directly from the spectra, we found that $2 / 3$ of the total flux originates in fields with inclinations below $45^{\circ}$, whereas LI08 give a ratio of 5 in favor of the transversal fields.

\subsection{Reliability of proxies in magnetic flux determination}

Since we were able to invert successfully most of the pixels because of the low noise level of our data, we have information about field strength, field inclination, and magnetic flux with some certainty. This allows us to test the validity of some of the approximations in LI08, who convert the total linear and circular polarization signal into their estimates of transversal and longitudinal flux. We calculated the longitudinal $\left(\Phi_{\mathrm{L}}\right)$ and transversal $\left(\Phi_{\mathrm{T}}\right)$ flux from the inversion results to be

$\Phi_{\mathrm{L}}=f \cdot B \cdot \cos \gamma \cdot A$

$\Phi_{\mathrm{T}}=f \cdot B \cdot \sin \gamma \cdot A$.

To a first order approximation, the circularly polarized Stokes $V$ signal should scale with $\Phi_{\mathrm{L}}$, whereas the linear polarization $L=\sqrt{Q^{2}+U^{2}}$ should scale with $\Phi_{\mathrm{T}}^{2}$ (Jefferies et al. 1989). From the observed profiles, we derived the maximal linear and circular polarization of the $1564.8 \mathrm{~nm}$ line $\left(L_{\max }\right.$ and $V_{\max }$, see 

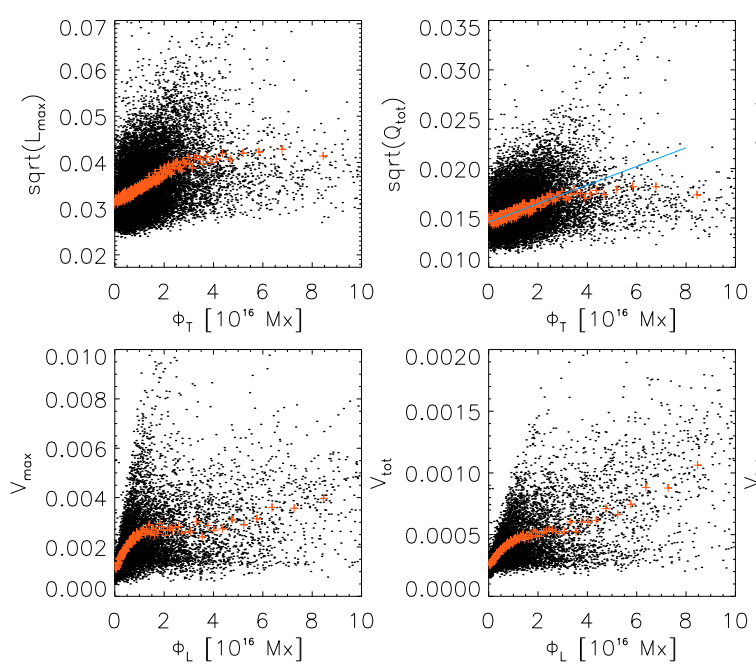

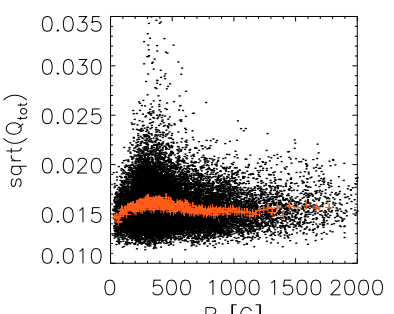

$B[G]$

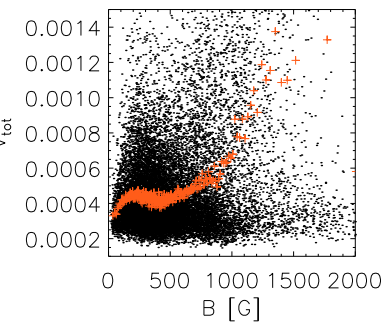

Fig. 12. Scatter plots of transversal and longitudinal flux versus maximal linear (left top) and circular polarization degree (left bottom), same versus total linear (middle top) and circular polarization (middle bottom), and scatter plot of field strength versus total linear (right top) and circular polarization (right bottom). Black: all data points; red: after binning. The blue line in the middle top panel is a linear fit to the binned data.
Sect. 2) and the total linear and circular polarization ( $L_{\text {tot }}$ and $V_{\text {tot }}$, see Sect. 3). In the derivation of $L_{\text {tot }}$, the noise in both $Q$ and $U$ enters. Following LI08, this contribution can be minimized by rotating the linear polarization signal to the "preferred azimuth frame", where the signal is concentrated in Stokes $Q$ (see Appendix B). In the following, we thus use $Q_{\text {tot }}$ (rotated) $=$ $\int|Q(\lambda)| \mathrm{d} \lambda$ after rotating the spectra, as a measure of the linear polarization instead of $L_{\text {tot }}$. Figure 12 shows scatter plots of $\Phi_{T}$ versus $\sqrt{Q_{\max }}$ and $\sqrt{Q_{\text {tot }}}$, and of $\Phi_{L}$ versus $V_{\max }$ and $V_{\text {tot. }}$. We also added a plot of field strength versus $\sqrt{Q_{\text {tot }}}$ and $V_{\text {tot }}$. It can be seen that there is on average a clear relation between $\Phi_{T}$ or $\Phi_{\mathrm{L}}$ and the corresponding quantities derived directly from the profiles (red points), but the scatter around this relation is large (there is around a 30\% difference between the mean and minimum or maximum values).

The polarization amplitudes and, hence also, the integrated polarization amplitudes measure the ratio of polarized to unpolarized light. Their absolute values strongly depend on the thermodynamical structure of the atmosphere and the structure resolution. This becomes even worse for VIS lines in the weak-field limit, where the field strength also changes the amplitudes of the polarization lobes, not only their location in wavelength. In Appendix B, we tested the derivation of calibration curves between transversal magnetic flux and $Q_{\text {tot }}$ based on various assumptions (constant inclination, constant flux, temperature variation). We found that only by varying the temperature stratification at constant magnetic flux, we can reproduce the scatter seen in Fig. 12. In total, the scatter in the relationship and the different calibration curves that can be obtained by, e.g., only varying the inclination by 10 degrees place the accuracy of the derivation of magnetic flux from proxies such as $Q_{\text {tot }}$ or $V_{\text {tot }}$ into some doubt.

However, apart from the observations with HINODE that favor a dominant fraction of horizontal fields, some simulation results also suggested that magnetic field are more horizontal than previously found. Schüssler \& Vögler (2008) and Steiner et al. (2008, ST08) reported the ratio of horizontal to vertical fields in their respective simulations, providing numbers from 2 to 6 , in accordance with the factor of 5 derived by LI08. This seems incompatible with the present investigation, but both simulations show that the ratio is strongly dependent on the height in the atmosphere. In their Fig. 1, ST08 show the field strengths as a function of height for two simulation runs (v10 = initial $10 \mathrm{G}$ vertical field, h20 = 20 G horizontal). At a height of around $200 \mathrm{~km}$, which is appropriate for the IR lines at $1565 \mathrm{~nm}$ used here, the ratio of v10 favors the longitudinal fields, $B_{\text {hor }} / B_{\text {ver }} \sim 0.82$. This is significantly closer to the ratio of $\Phi_{\gamma>45^{\circ}} / \Phi_{\gamma<45^{\circ}}=0.42$ than the factor of 5 that ST08 derived after synthesizing $630 \mathrm{~nm}$ spectra of their simulation and analyzing them in the same way as LI08.

\subsection{Vertical unsigned flux density}

Various authors have derived the average unsigned flux density of the vertical magnetic fields from Zeeman-sensitive spectral lines (Keller et al. 1994; Lites 2002; Domínguez Cerdeña et al. 2003; Khomenko et al. 2005a; Domínguez Cerdeña et al. 2006a,b; Orozco Suárez et al. 2007; Martínez González et al. 2008c; Lites et al. 2008; Carroll \& Kopf 2008). In the older literature, magnetic field proxies such as the Stokes $V$ amplitude or integrated area were used, and more recently an inversion of spectra. The obtained values for $\left\langle B_{z}\right\rangle$ have significant scatter, depending on the observations used or the analysis method employed, but in general yield values between 5 and $30 \mathrm{G}$. In addition, LI08 measured a transversal magnetic flux of around $50 \mathrm{G}$. Observations using the Hanle effect yielded significantly higher values of around $100 \mathrm{G}$ (e.g., Trujillo Bueno et al. 2004).

Figure 13 shows the distribution of $\left\langle B_{z}\right\rangle$ in our observations, derived from the longitudinal flux after division by the area $A$ (cf. Eq. (2)); the bin size was $0.5 \mathrm{G}$. The distribution shows a maximum near $2 \mathrm{G}$, but is otherwise in good agreement with a monotonically increasing probability at weaker fluxes (solid red line, exponential decay law). The difference between the exponential decay and the observed distribution for $\Phi<2 \mathrm{G}$ only covers a small area of far less than $10 \%$ relative frequency (red filled area in the upper panel of Fig. 13 from 0 to $2 \mathrm{G}$, see the inlet). In the double-logarithmic display in the lower half, we displayed the first 7 histogram points with crosses. It can be clearly seen that from high fluxes up to the maximum probability value the points closely follow an exponential law; only the first four points corresponding to the weakest fluxes below $2 \mathrm{G}$ deviate from the principal trend. Since the noise imposes a hard threshold on the detection of the lowest magnetic fluxes, we believe that the occurrence of the maximum is fully artificial. Very weak fluxes produce increasingly weaker polarization signals that can escape detection completely or are eventually rejected by the inversion threshold. Pietarila Graham et al. (2009) claimed that the distribution of the magnetic flux density cannot be determined from observations because of the peaked appearance of the probability distributions as in Fig. 13. However, given the small deviation between the observed distribution and an 

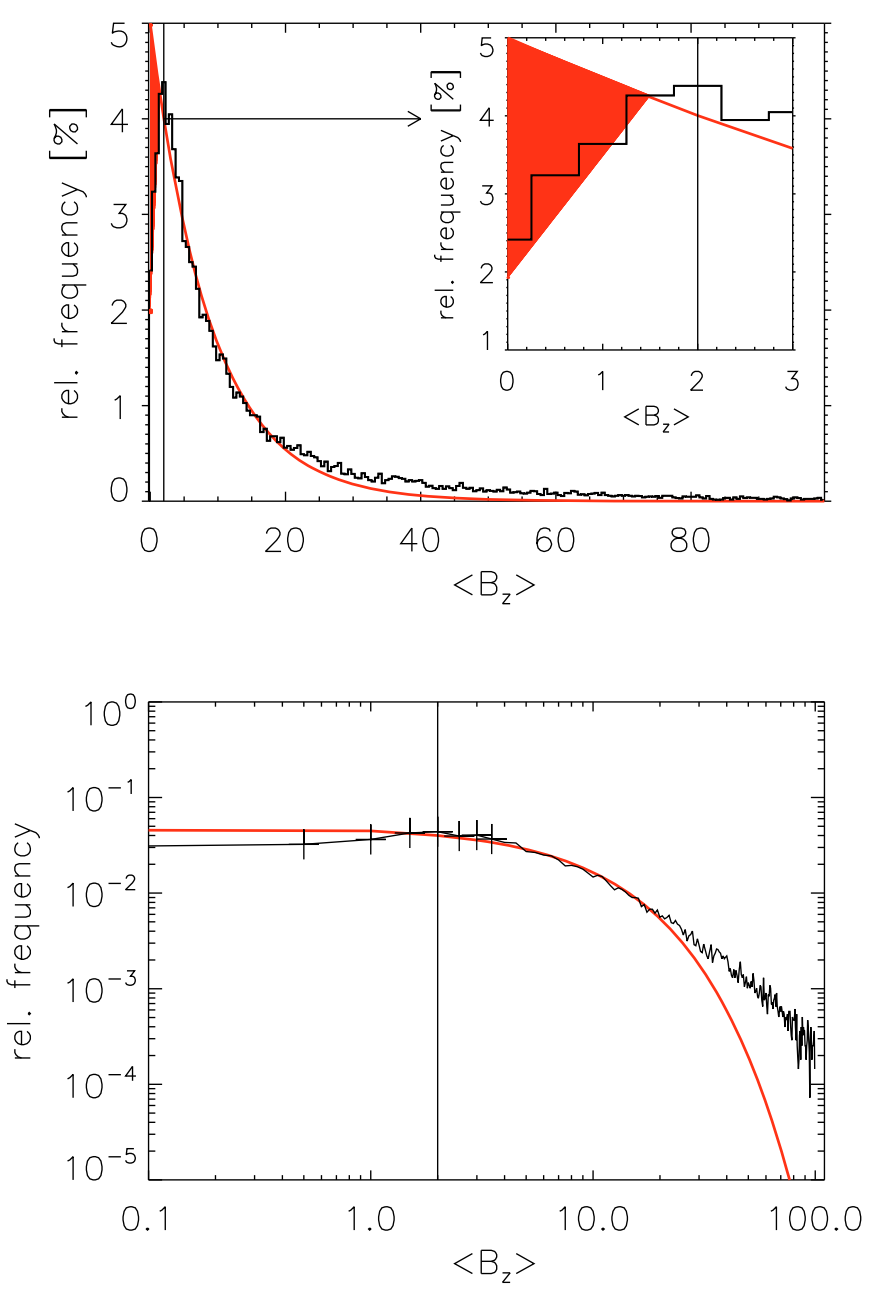

Fig. 13. Histograms of vertical flux density in linear (top) and logarithmic scale (bottom). The vertical lines mark 2 G. Black and red lines show the observations and an exponential decay law. The inlet in the upper panel shows a magnification of the low flux range.

exponential decay law predicted by MHD simulations, we propose that the probability distribution function of magnetic flux can be derived from observations when one considers that the weakest fluxes must escape detection because of the noise level.

Using the strongly Zeeman-sensitive IR lines at $1.56 \mu \mathrm{m}$, it is possible to disentangle the field strength $B$ on the part of the surface that is covered by fields from the area filling fraction $f$ of the magnetic structure that is almost certainly unresolved. This makes an important difference to the calculation of the magnetic flux density, compared with the spectral lines at $630 \mathrm{~nm}$ used by the HINODE spectropolarimeter and in the work of Pietarila Graham et al. (2009). The $630 \mathrm{~nm}$ lines are in the weak-field limit for the weak magnetic fields of the quiet Sun, i.e., they are sensitive to a strong interplay between thermodynamics and magnetic field properties, as also demonstrated in Appendix B. It is more difficult to determine $B$ and $f$ accurately for these lines, even if one carefully considers the thermodynamics (Martínez González et al. 2006b).

Normalizing the observed magnetic flux to the complete FOV, including the locations with no significant polarization signal, the average values for the total, longitudinal, and transversal flux using Eqs. (2) and (3) are $22 \mathrm{G}, 16 \mathrm{G}$, and $11 \mathrm{G}$, respectively. If one only considers the area of actually observed polarization signal, the corresponding values are $26 \mathrm{G}, 20 \mathrm{G}$, and
$13 \mathrm{G}$. The values are at the upper range of Zeeman-based measurements. As mentioned by, e.g., Pietarila Graham et al. (2009), the cancellation of Stokes $V$ signals due to unresolved opposite polarities inside a resolution element causes an underestimation of the true amount of magnetic flux. Our observations with a $0.5 \times 0$ '. 37 sampling are prone to this. The average flux densities can thus only be taken as a lower limit to the total magnetic flux, but we believe that they represent a rather solid limit to the total magnetic flux observed at the $1^{\prime \prime}$ resolution achieved.

\section{Summary and conclusions}

We have analysed two long-integration data sets of quiet Sun regions obtained under extremely good seeing conditions with the TIP at $1.56 \mu \mathrm{m}$. We have found that the data allow us to study the weak horizontal internetwork fields that appeared prominently in HINODE observations recently (OR07 and LI08). Because of the lower noise level than for HINODE data and the higher sensitivity of the IR lines, we were able to invert the spectra with SIR and avoid using strong approximations in the derivation of magnetic field properties. We found that the more inclined/“horizontal" fields $\left(\gamma>45^{\circ}\right)$ are generally weaker $(<500 \mathrm{G})$ and contain in total less magnetic flux than the less inclined/"vertical" fields. Since the data properties (spatial scales of observed structures, pixels with significant polarization signal) and the inversion results (magnetic filling fraction, relation to granules/IGLs) seem to agree roughly with the HINODE data and their analysis in OR07 and LI08, we ascribe the reversal in the ratio of transversal to longitudinal fields (HINODE data: 5, IR data: 0.42) to: a) the different formation height of the spectral lines, and b) the analysis methods, i.e., either inversions using the ME approximation or a consistent treatment of thermodynamics (SIR), or calibration from integrated polarization signal to magnetic flux. We conclude that the issue would be investigated most effectively with simultaneous IR/VIS data, since simulations predict a strong dependence of the ratio on geometrical height (Steiner et al. 2008). Although the existence of a significant fraction of inclined magnetic fields is confirmed by the present IR observations, their influence on the dynamics of the solar atmosphere remains unclear. They do not seem to affect the granulation pattern, but could contribute to chromospheric heating as discussed in Ishikawa \& Tsuneta (2009). This issue could be addressed by a combination of repeated spectropolarimetry of photospheric fields with chromospheric diagnostics.

Acknowledgements. The VTT is operated by the Kiepenheuer-Institut für Sonnenphysik (KIS) at the Spanish Observatorio del Teide of the Instituto de Astrofísica de Canarias (IAC). R.R. acknowledges support by the Deutsche Forschungsgemeinschaft under grant SCHM 1168/8-2. C.B. thanks M. Collados for being the only co-observer to show up during the campaign. We thank O. Steiner (KIS) for making the $\mathrm{CO}^{5} \mathrm{BOLD}$ simulation data available to us.

\section{References}

Beck, C. 2006, Ph.D. Thesis, Albert-Ludwigs-University, Freiburg

Beck, C., Schlichenmaier, R., Collados, M., Bellot Rubio, L., \& Kentischer, T. 2005a, A\&A, 443, 1047

Beck, C., Schmidt, W., Kentischer, T., \& Elmore, D. 2005b, A\&A, 437, 1159

Beck, C., Bellot Rubio, L. R., Schlichenmaier, R., \& Sütterlin, P. 2007, A\&A, 472, 607

Bellot Rubio, L. R. 2003, Kiepenheuer Institut für Sonnenphysik, Freiburg Bellot Rubio, L. R., \& Collados, M. 2003, A\&A, 406, 357

Bellot Rubio, L. R., Ruiz Cobo, B., \& Collados, M. 2000, ApJ, 535, 475

Cabrera Solana, D., Bellot Rubio, L. R., \& del Toro Iniesta, J. C. 2005, A\&A, 439,687 
Carroll, T. A., \& Kopf, M. 2008, A\&A, 481, L37

Collados, M., Lagg, A., Díaz Garcí A. J. J., et al. 2007, in The Physics of Chromospheric Plasmas, ed. P. Heinzel, I. Dorotovič, \& R. J. Rutten, ASP Conf. Ser., 368, 611

de Wijn, A. G., Lites, B. W., Berger, T. E., et al. 2008, ApJ, 684, 1469

Domínguez Cerdeña, I., Sánchez Almeida, J., \& Kneer, F. 2003, A\&A, 407, 741

Domínguez Cerdeña, I., Almeida, J. S., \& Kneer, F. 2006a, ApJ, 646, 1421

Domínguez Cerdeña, I., Sánchez Almeida, J., \& Kneer, F. 2006b, ApJ, 636, 496

Gingerich, O., Noyes, R. W., Kalkofen, W., \& Cuny, Y. 1971, Sol. Phys., 18, 347 Harvey, J. W., Branston, D., Henney, C. J., \& Keller, C. U. 2007, ApJ, 659, L177

Ishikawa, R., \& Tsuneta, S. 2009, A\&A, 495, 607

Ishikawa, R., Tsuneta, S., Ichimoto, K., et al. 2008, A\&A, 481, L25

Jefferies, J., Lites, B. W., \& Skumanich, A. 1989, ApJ, 343, 920

Keller, C. U., Deubner, F.-L., Egger, U., Fleck, B., \& Povel, H. P. 1994, A\&A, 286, 626

Kentischer, T. J., Schmidt, W., Sigwarth, M., \& von Uexküll, M. 1998, A\&A, 340,569

Khomenko, E. V., Collados, M., Solanki, S. K., Lagg, A., \& Trujillo Bueno, J. 2003, A\&A, 408, 1115

Khomenko, E. V., Martínez González, M. J., Collados, M., et al. 2005a, A\&A, 436, L27

Khomenko, E. V., Shelyag, S., Solanki, S. K., \& Vögler, A. 2005b, A\&A, 442, 1059

Kosugi, T., Matsuzaki, K., Sakao, T., et al. 2007, Sol. Phys., 243, 3

Lin, H. 1995, ApJ, 446, 421

Lites, B. W. 2002, ApJ, 573, 431

Lites, B. W., \& Socas-Navarro, H. 2004, ApJ, 613, 600

Lites, B. W., Leka, K. D., Skumanich, A., Martinez Pillet, V., \& Shimizu, T. 1996, ApJ, 460, 1019

Lites, B. W., Kubo, M., Socas-Navarro, H., et al. 2008, ApJ, 672, 1237

Martínez Pillet, V., Collados, M., Sánchez Almeida, J., et al. 1999, in High Resolution Solar Physics: Theory, Observations, and Techniques, ASP Conf. Ser., 183, 264
Martínez González, M. J., Collados, M., \& Ruiz Cobo, B. 2006a, in Astronomical Society of the Pacific Conference Series, ed. R. Casini, \& B. W. Lites, ASP Conf. Ser., 358, 36

Martínez González, M. J., Collados, M., \& Ruiz Cobo, B. 2006b, A\&A, 456, 1159

Martínez González, M. J., Asensio Ramos, A., Carroll, T. A., et al. 2008a, A\&A, 486, 637

Martínez González, M. J., Asensio Ramos, A., López Ariste, A., \& Manso Sainz, R. 2008b, A\&A, 479, 229

Martínez González, M. J., Collados, M., Ruiz Cobo, B., \& Beck, C. 2008c, A\&A, 477, 953

Orozco Suárez, D., Bellot Rubio, L., del Toro Iniesta, J., et al. 2007, ApJ, 670, L61

Pietarila Graham, J., Danilovic, S., \& Schuessler, M. 2009, ApJ, 693, 1728

Rezaei, R., Schlichenmaier, R., Beck, C. A. R., Bruls, J. H. M. J., \& Schmidt, W. 2007, A\&A, 466, 1131

Ruiz Cobo, B., \& del Toro Iniesta, J. C. 1992, ApJ, 398, 375

Sánchez Almeida, J., \& Lites, B. W. 2000, ApJ, 532, 1215

Schaffenberger, W., Wedemeyer-Böhm, S., Steiner, O., \& Freytag, B. 2005, in Chromospheric and Coronal Magnetic Fields, ed. D. E. Innes, A. Lagg, \& S. A. Solanki, ESA SP, 596

Schaffenberger, W., Wedemeyer-Böhm, S., Steiner, O., \& Freytag, B. 2006, in Solar MHD Theory and Observations: A High Spatial Resolution Perspective, ed. J. Leibacher, R. F. Stein, \& H. Uitenbroek, ASP Conf. Ser., 354, 345

Schlichenmaier, R., \& Collados, M. 2002, A\&A, 381, 668

Schüssler, M., \& Vögler, A. 2008, A\&A, 481, L5

Sheminova, V. A. 2009, Sol. Phys., 254, 29

Socas-Navarro, H., \& Sánchez Almeida, J. 2003, ApJ, 593, 581

Socas-Navarro, H., \& Lites, B. W. 2004, ApJ, 616, 587

Steiner, O., Rezaei, R., Schaffenberger, W., \& Wedemeyer-Böhm, S. 2008, ApJ, 680, L85

Trujillo Bueno, J., Shchukina, N., \& Asensio Ramos, A. 2004, Nature, 430, 326 Vögler, A., Shelyag, S., Schüssler, M., et al. 2005, A\&A, 429, 335

von der Lühe, O., Soltau, D., Berkefeld, T., \& Schelenz, T. 2003, in Proc. SPIE, ed. by Stephen L. Keil, Sergey V. Avakyan, 4853, 187 


\section{Appendix A: Examples of inverted spectra}

Figures A.1 and A.2 show several profiles taken from the first and second long-integration observation of 2008 May 21 (Op. 001 and Op. 005), respectively. The positions of the profiles are marked by consecutive numbers in Fig. 1. The profiles shown were selected to have a small polarization degree that in some cases was barely sufficient to meet the inversion threshold (e.g., profiles Nos. 6 and 7). Below the spectra, the temperature stratifications used in the generation of the best-fit spectra are shown. With 3 nodes in temperature, the SIR code can use a parabola for changing the stratification; the parabola shape appears quite prominent for many of the locations. We note, however, that the IR lines at $1.56 \mu \mathrm{m}$ are not sensitive to the temperature in the atmosphere above $\log \tau \sim-1.5$ (Cabrera Solana et al. 2005). Only one profile corresponds to a $\mathrm{kG}$ field (Fig. A.2, top middle, No. 8). Figure A.3 shows the polarization degree of $1564.8 \mathrm{~nm}$ for all profiles of the previous figures. Profile No. 9 exceeds the inversion threshold of 0.001 of $I_{\mathrm{c}}$ near $+750 \mathrm{~m} \AA$ with a spike that is presumably not of solar origin, but is instead noise in the Stokes $U$ profile. The final rejection threshold of 0.0014 is, however, only reached by signals clearly related to the Zeeman effect (multiple double or triple lobes).

Reliability of the inversion results. As discussed in Sect. 3, we used a constant value for the magnetic field strength $(B)$, inclination $(\gamma)$, azimuth $(\psi)$, and the LOS velocity. This inversion setup cannot reproduce the antisymmetric Stokes $Q$ or $U$ or symmetric Stokes $V$ profiles, which would require gradients in the magnetic field strength and the velocities along the LOS. The inversion was initialized with the same model atmosphere on all pixels ( $B=0.9 \mathrm{kG}, \psi=65 \mathrm{deg}$ ), only the inclination being modified to 10 or $170 \mathrm{deg}$ depending on the polarity. In the inversion process, the equal weight used for $Q U V$ in the calculation of $\chi^{2}$ naturally favors the component of higher polarization signal. For example, in profile no. 5 in Fig. A. 1 the $Q$ and $U$ signals are larger than the $V$ signal by almost an order of magnitude, leading to a better fit quality for $Q$ and $U$ than for $V$. In polarimetric data of low $\mathrm{S} / \mathrm{N}$, a difference of this order usually implies that the weaker signal is not seen at all.

SIR calculates an error estimate for the free fit parameters using the diagonal elements of the covariance matrix, expressed by the response functions (Bellot Rubio et al. 2000; Bellot Rubio 2003). The error estimate depends on the number of degrees of freedom in each variable; for parameters constant with optical depth thus a single value is returned. The error estimate, however, provides only information about the reliability of the bestfit solution for the corresponding $\chi^{2}$-minimum inside the chosen inversion setup. The estimated errors in the inversion of the profiles shown in Figs. 4, A.1, and A.2 are noted on the Stokes I panel for the Fe I $1565.2 \mathrm{~nm}$ line. The average uncertainties in the calculated magnetic field strength and inclination angle given by SIR are $\pm 50 \mathrm{G}$ and $\pm 10 \mathrm{deg}$, respectively. The values agree with a previous error estimate in Beck (2006) derived from a direct analysis of the profile shape of the $1.56 \mu \mathrm{m}$ lines (Table 3.2 on p. $47 ; \delta B \sim 50 \mathrm{G}$ and $\delta \theta \sim 5 \mathrm{deg}$ ).

\section{Appendix B: Calibration of $L_{\text {tot }}$ to transversal flux}

We tried to follow the procedure described in LI08 to calibrate the linear polarization signal into a transversal magnetic flux estimate that is independent of the inversion results. To reduce the influence of noise, LI08 first determine the "preferred azimuth frame", where the linear polarization signal is concentrated in Stokes $Q$. To achieve this, we determined the azimuth angle from the ratio of $U$ to $Q$, and rotated the spectra correspondingly to maximize the Stokes $Q$ signal. The scatter plot in Fig. B.1 compares the previously used total linear polarization, $L_{\mathrm{tot}}=\int \sqrt{Q^{2}+U^{2}}(\lambda) \mathrm{d} \lambda$, with the corresponding $Q_{\text {tot }}($ rotated $)=\int|Q(\lambda)| \mathrm{d} \lambda$ as a measure of the linear polarization. The rotation of the spectra reduces the noise contribution by a constant amount, but the old and new values otherwise have a linear relationship with a slope close to unity.

We then averaged the rotated $Q$ spectra over all spatial positions exceeding the polarization threshold for the inversion. The average Stokes $Q$ spectrum was used as a spectral mask by LI08, but unfortunately their method fails for the infrared lines. The wavelengths around the line core have negative values in the average $Q$ profile (Fig. B.2), which prevents to use it in the same way as in LI08. We thus used $Q_{\text {tot }}$ (rotated) as defined above instead, which we understand to be equivalent to the approach of LI08 despite not using a (somewhat arbitrary) spectral mask.

The plot of $Q_{\text {tot }}$ (rotated) versus transversal flux (Fig. 12, middle upper panel) showed considerable scatter that places the use of a single calibration curve in doubt. We thus not only tried to obtain a calibration curve, but also to quantify the effect of various parameters on the obtained relation. The upper part of Fig. B.3 shows calibration curves of $Q_{\text {tot }}$ versus field strength for different field inclinations $\gamma$. The uppermost curve for $\gamma=90^{\circ}$ corresponds to the one used by LI08. With the assumption that the field inclination does not necessary equal $90^{\circ}$, one already finds that one and the same value of $Q_{\text {tot }}$ can be obtained for a range of around $200-550 \mathrm{G}$ in field strength. The same effect is shown in the middle part, where the magnetic flux, $\Phi=B \sin \gamma$, was kept constant at $1.8 \times 10^{16} \mathrm{Mx}, B$ was varied, and $\gamma$ was derived accordingly from $\gamma=\arcsin (\Phi / B)$. Again a range of around 200-500 G in $B$ corresponds to the same value of $Q_{\text {tot }}$. As a final test, we chose to investigate the influence of the temperature stratification on the resulting $Q_{\text {tot }}$-value. We retained the magnetic flux, field strength and field inclination constant at $\left(1.8 \times 10^{16} \mathrm{Mx}, 20 \mathrm{G}, 75^{\circ}\right)$, and synthesized spectra for different temperature stratifications. We used 10000 temperature stratifications that were derived for the magnetic component in the inversion, and thus can be taken to be an estimate of the range of temperatures expected in the quiet Sun. The histogram of the resulting $Q_{\text {tot }}$-values is displayed in the bottom part of Fig. B.3. The value of $\sqrt{Q_{\text {tot }}}$ ranges from nearly zero up to 0.01 , which also roughly corresponds to the scatter in $\sqrt{Q_{\text {tot }}}$ in Fig. 12. We thus conclude that the largest contribution to the scatter comes from temperature effects. We remark that we used a magnetic filling factor of unity in all calculations. Any additional variation in the filling factor due to unresolved magnetic structures would increase the scatter in $Q_{\text {tot }}$ even more.

We conclude that the usage of a calibration curve for a derivation of transversal magnetic flux from $L_{\mathrm{tot}}$ or $Q_{\mathrm{tot}}$, regardless of the exact calculation of the wavelength integrated quantities, is not reliable for a solid estimate, mainly because the strong influence of the thermodynamical state of the atmosphere on the weak polarization signals. 
C. Beck and R. Rezaei: Magnetic flux in the internetwork, Online Material p 2

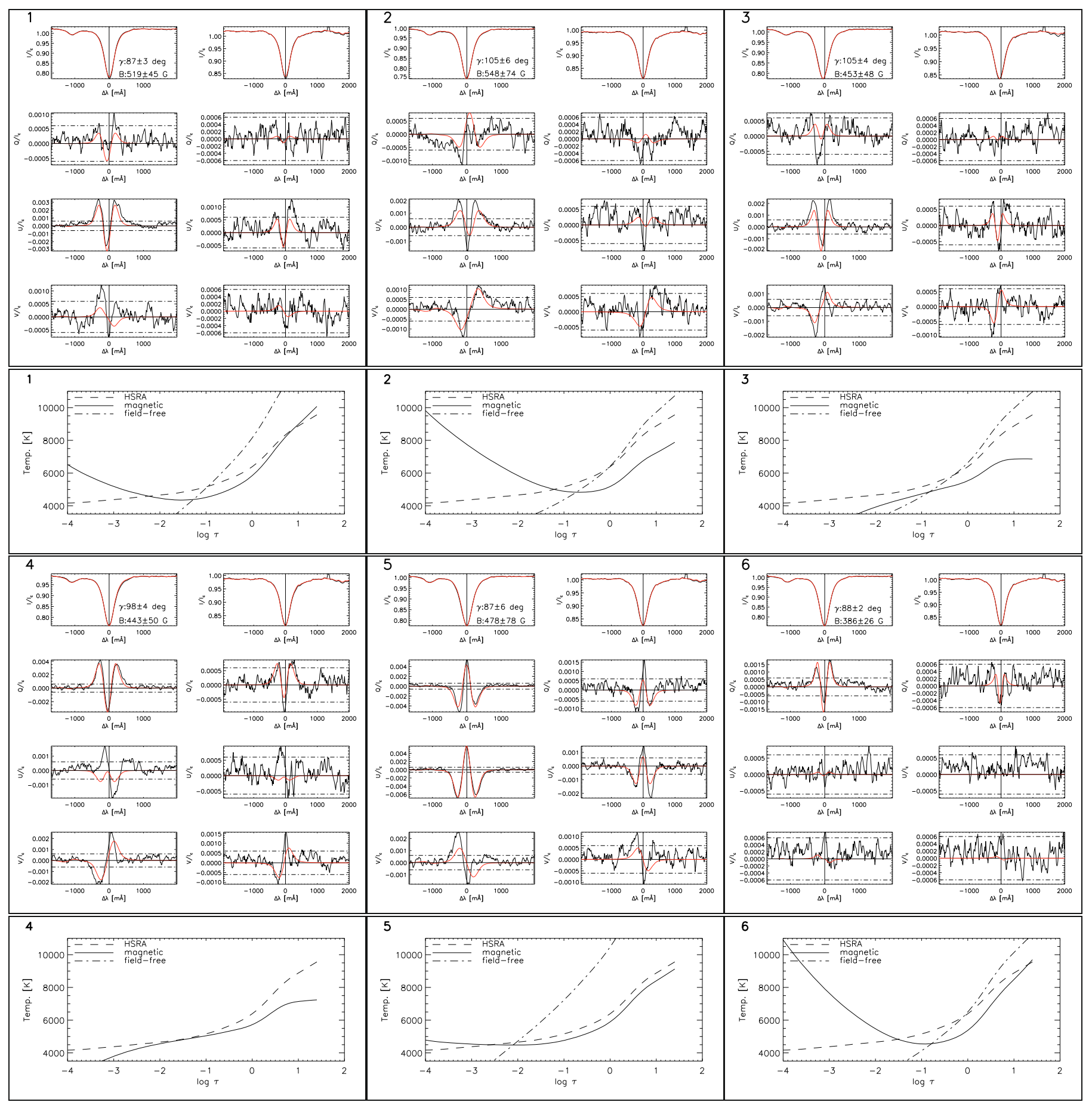

Fig. A.1. Examples of observed (black line) and best-fit spectra (red) in Op. 001 (1st and 3rd row). The dash-dotted horizontal lines in QUV indicate three times the rms noise level, and the solid horizontal line the zero level. The vertical solid line denotes the rest wavelength. The 2 nd and 4th row show the corresponding temperature stratifications of the magnetic component (solid), the field-free component (dash-dotted), and the HSRA atmosphere that is used as initial model (dashed). Field strength and LOS inclination and their respective errors are given in the plot of Stokes $I$ of $1564.8 \mathrm{~nm}$ (upper left in each panel); the number of each profile is given in the upper left corner of each panel. 
C. Beck and R. Rezaei: Magnetic flux in the internetwork, Online Material p 3

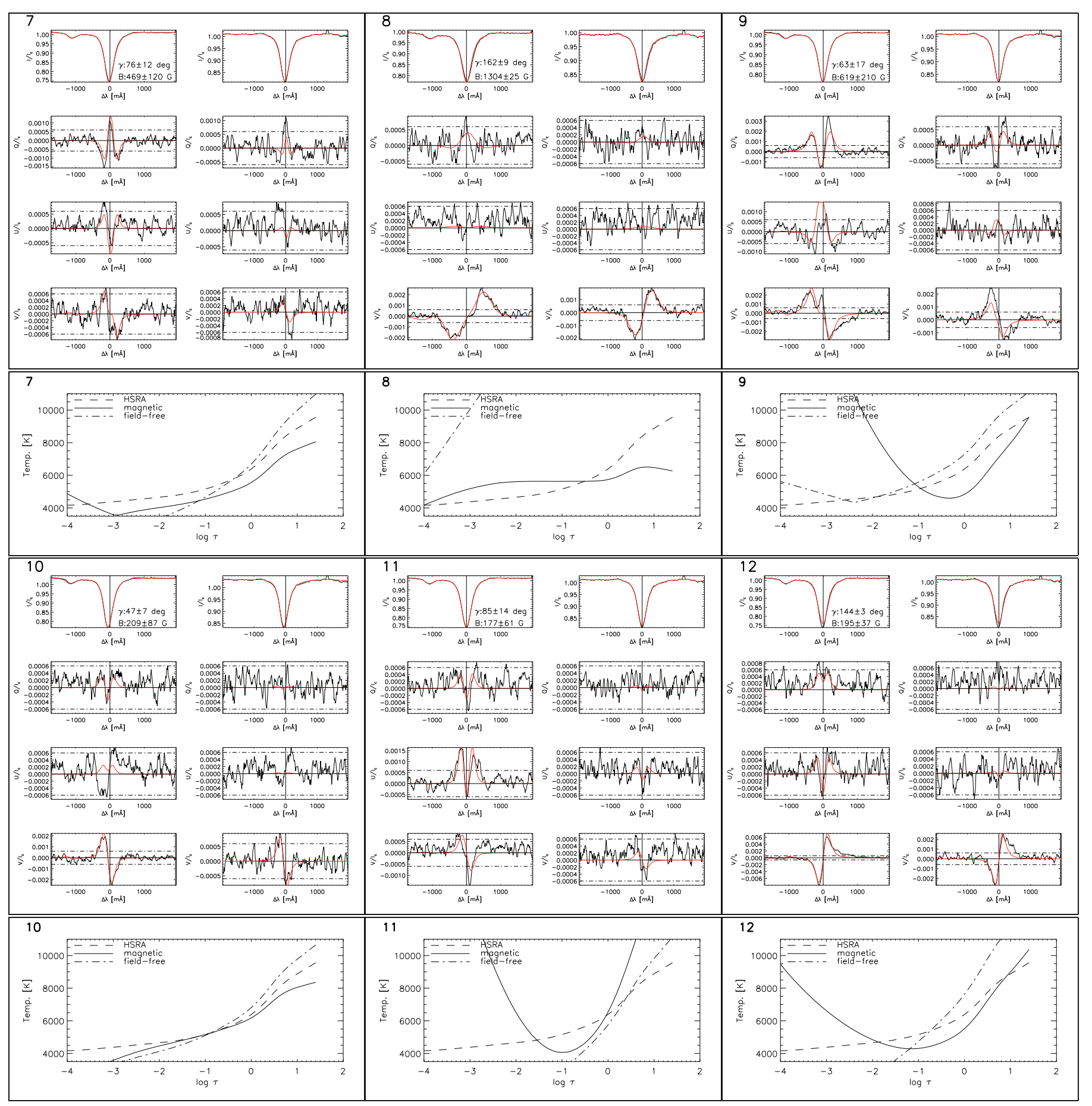

Fig. A.2. Same as Fig. A.1 for TIP Op. 005. 
C. Beck and R. Rezaei: Magnetic flux in the internetwork, Online Material p 4

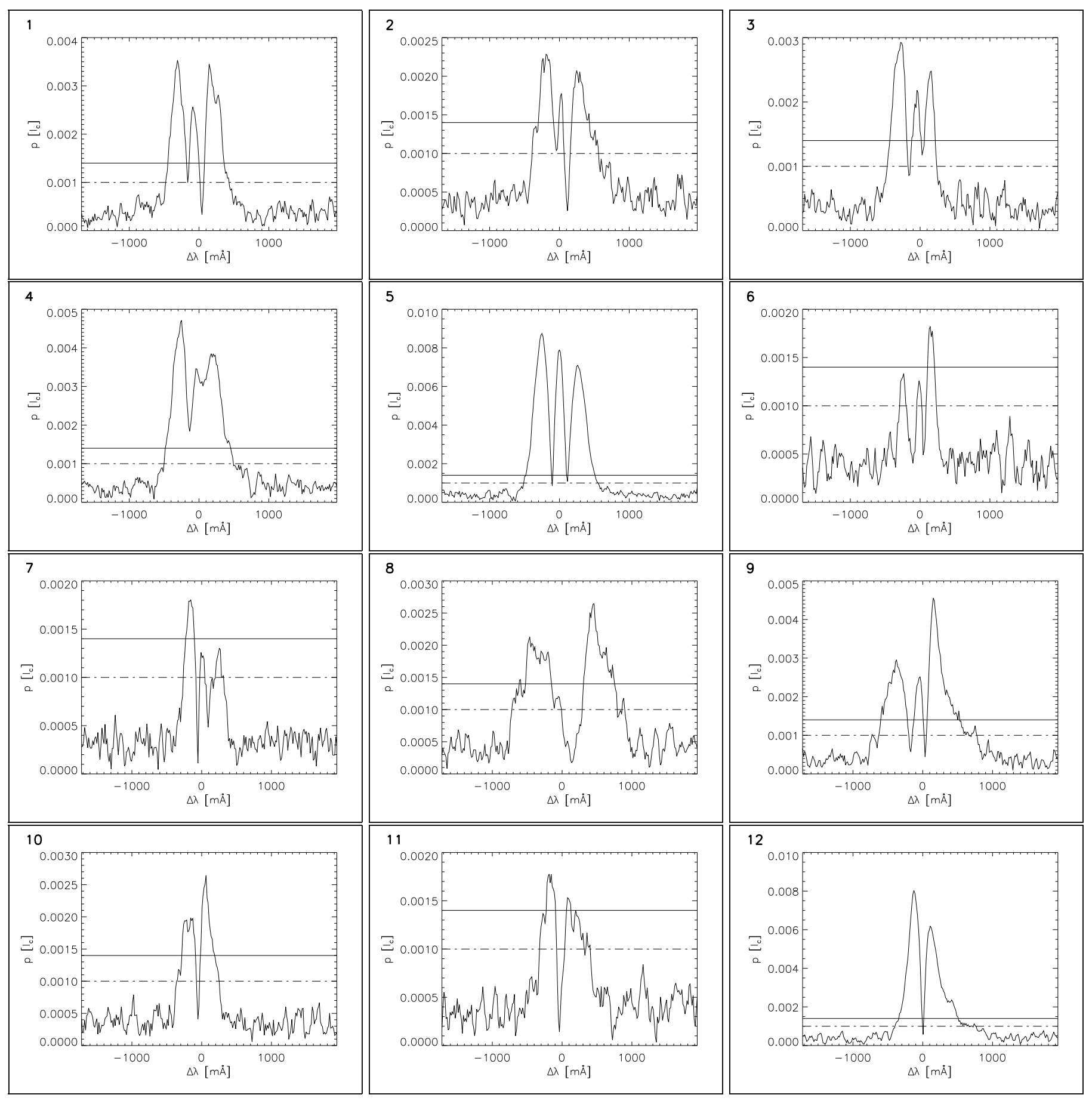

Fig. A.3. Polarization degree of $1564.8 \mathrm{~nm}$ for the profiles shown in Figs. A.1 (upper two rows) and A.2 (lower two rows). The dash-dotted and solid horizontal lines denote the inversion and final rejection threshold, respectively. 
C. Beck and R. Rezaei: Magnetic flux in the internetwork, Online Material p 5

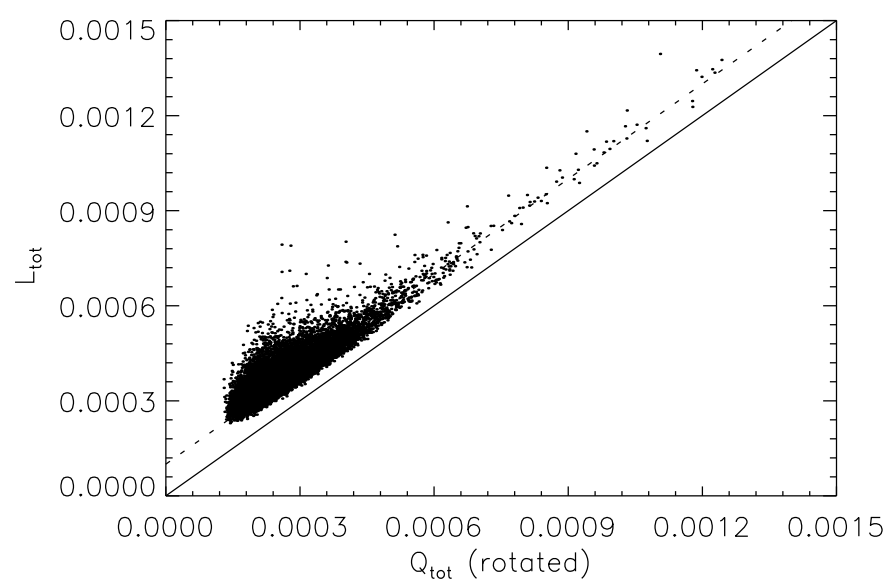

Fig. B.1. Scatter plot of the integrated Stokes $Q$ signal in the preferred reference frame versus the total linear polarization without rotation. Solid line: unity slope; dashed line: unity slope with an offset of 0.0001 .

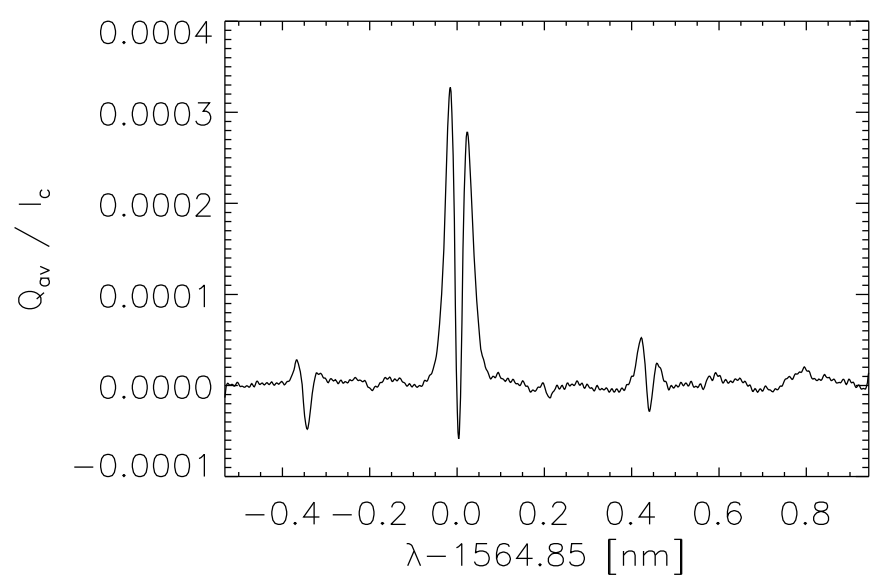

Fig. B.2. The average Stokes $Q$ profile.
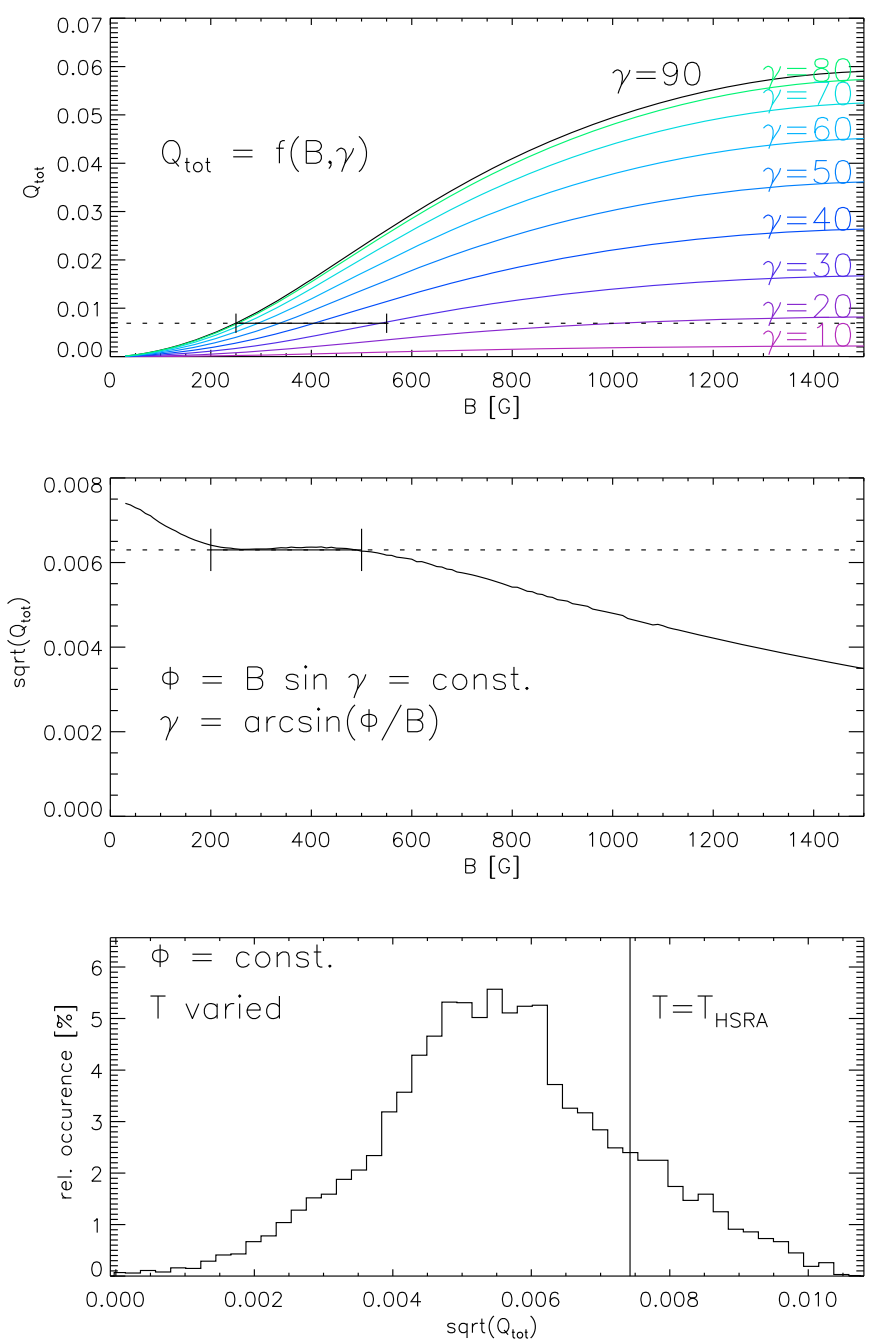

Fig. B.3. Top: calibration curves from $Q_{\text {tot }}$ into field strength $B$ for field inclinations $\gamma$ from 10 to $90 \mathrm{deg}$ (bottom to top). The horizontal dotted line is at $Q_{\mathrm{tot}}=0.007$; the solid part of it denotes a range in $B$ that gives the same $Q_{\text {tot }}$ at different $\gamma$. Middle: $\sqrt{Q_{\text {tot }}}$ versus field strength for constant magnetic flux. Dotted line and solid part as above for $\sqrt{Q_{\mathrm{tot}}}=0.0063$. Bottom: histogram of $\sqrt{Q_{\mathrm{tot}}}$ for constant flux but varying temperature stratifications $T$. The vertical line denotes the value resulting from the HSRA atmosphere model. 